\title{
Oscillatory spatially periodic weakly nonlinear gravity waves on deep water
}

\author{
By JUAN A. ZUFIRIA \\ Applied Mathematics, California Institute of Technology, Pasadena, CA 91125, USA
}

(Received 23 April 1987 and in revised form 24 August 1987)

A weakly nonlinear Hamiltonian model is derived from the exact water wave equations to study the time evolution of spatially periodic wavetrains. The model assumes that the spatial spectrum of the wavetrain is formed by only three free waves, i.e. a carrier and two side bands. The model has the same symmetries and invariances as the exact equations. As a result, it is found that not only the permanent form travelling waves and their stability are important in describing the time evolution of the waves, but also a new kind of family of solutions which has two basic frequencies plays a crucial role in the dynamics of the waves. It is also shown that three is the minimum number of free waves which is necessary to have chaotic behaviour of water waves.

\section{Introduction}

For more than a century it has been known that irrotational surface waves exist which travel with permanent form on water. But, until Lighthill (1965) and Benjamin \& Feir (1967) discovered that these waves are unstable to modulation perturbations, not much attention was paid to the stability problem. Benjamin \& Feir's work predicted the initial growth rate of the instabilities, but left open the question of the long-time evolution of these unstable wavetrains. After this discovery, great interest was born within the scientific community in predicting the long-time behaviour of unstable water waves.

Initially, different authors suggested possibilities which ranged from the complete breakdown of the wavetrain with equipartition of the energy among all the modes, to a train of stable soliton envelopes. For a review of the history see Yuen \& Lake (1982).

But experiments in a tank performed by Lake et al. (1977) showed that unstable modulations grow in time, reach a maximum and then subside. The wavetrain undergoes an unsteady series of modulation-demodulation cycles which are known as the Fermi-Pasta-Ulam (1955) recurrence phenomenon.

Zakharov (1968) (and later Hasimoto \& Ono 1972) showed that, when the wavenumber variation is small, the governing equations of the process can be reduced to the nonlinear Schrödinger equation. This equation was used by Lake et al. (1977) to confirm theoretically the results that they found in the experiments.

Because of the limitations in the length of the tank and the dissipation due to local breaking and viscosity, the experiments performed by Lake et al. showed only the first modulation-demodulation period. Since then, most of the work trying to understand the long-time evolution of trains of waves has been theoretical. This theoretical work has been basically focused on the study of the properties of the nonlinear Schrödinger equation and of a discrete version of the Zakharov integral 
equation (Zakharov 1968; also see Yuen \& Lake 1982). The discrete version of the Zakharov integral equation is obtained by assuming that the spatial spectrum of the waves can be decomposed by a $\delta$-function representation into a discrete set of modulated waves. The equation is then reduced to a system of complex ordinary differential equations which describe the interactions of the different free modes during the time evolution.

The exact irrotational water wave equations are conservative, and Zakharov (1968) proved that a Hamiltonian exists. Hence, the system is a continuous Hamiltonian system. On the other hand, the discrete Zakharov equations form a non-conservative but phase-space volume preserving system. Caponi, Saffman \& Yuen (1982) have shown that the system may present periodic, recurrent and chaotic evolution. They found that in order to have chaotic behaviour a minimum of seven modes (i.e. 14 real ODEs) in the discrete spatial spectrum need to be considered. Also, Yuen (1984) found that the results depended very greatly on the number of modes used in the computations.

In accordance with the symmetries of the water wave problem, besides the trivial conversation of mass, there are two basic conserved quantities which are the energy and the horizontal momentum of the waves. Therefore, one could expect three degrees of freedom (i.e. $6 \mathrm{ODEs}$ ) to be enough to have a non-integrable system, i.e. for the possibility for the system to show irregular or chaotic time evolution. This suggests that the symmetries of the discrete Zakharov equations do not agree with those of the original problem.

All these facts motivate us to derive some evolution equations which have the same symmetries as the original water wave problem. In the present work we are going to derive a set of weakly nonlinear equations to study the long-time evolution of periodic trains of waves. Even though the procedure to derive the equations is independent of the number of modes, we will concentrate our study on the case of only three modes on water of infinite depth. This case, besides being the simplest one which can lead to irregular behaviour, is already rich enough to show many important properties of the dynamics of water waves.

Our main goal is to give a qualitative description of the dynamics of water waves by using the theory of discrete Hamiltonian systems. It will not be our concern to give exact values of the periods and amplitudes of the waves versus time. We will give a description of the different kinds of motions, and an explanation of their origin. We will show where and why chaotic behaviour can be expected in water waves.

In $\S 2$, we obtain the three-wave model from the full water wave equations. In $\S 3$, the steady travelling waves of the system and their stability properties are analysed. In this section, we also reduce the number of degrees of freedom of our system by using the symmetries of the problem. New kinds of solutions of the water wave problem are computed in $\S 4$. These solutions correspond to waves which have two basic frequencies. It is shown that the stability of these new solutions plays a very important role in the dynamics of the system. Finally in $\$ 5$, we reduce the analysis of our system to the study of the properties of an area preserving map. This area preserving map shows the different kinds of motions which can be expected from our system. 


\section{Model equation}

Consider two-dimensional irrotational surface waves on water of infinite depth. Zakharov (1968) and Broer (1974) have shown that, when the shape of the wave $\eta(x, t)$ and the value of the velocity potential $\Phi(x, y, t)$ evaluated on the surface of the wave $\psi(x, t)=\Phi(x, \eta(x, t), t)$ are used to describe a wave, the evolution equations for the wave system can be written with the following Hamiltonian structure

$$
\frac{\partial \eta}{\partial t}=\frac{\delta \mathscr{H}}{\delta \psi}, \quad \frac{\partial \psi}{\partial t}=-\frac{\delta \mathscr{H}}{\delta \eta},
$$

where $\delta$ represents a functional derivative (see Goldstein 1980 ), and $\mathscr{H}$ is the total energy of the waves. When periodic waves are considered the lengthscale can be chosen such that the period of the wave is $2 \pi$. With this choice of the lengthscale, $\mathscr{H}$ is given by

$$
\mathscr{H}=\int_{0}^{2 \pi} H(\eta, \psi) \mathrm{d} x
$$

where $H$ is the energy density. For gravity waves we have

$$
H=\frac{1}{2} \int_{-\infty}^{\eta}(\nabla \Phi)^{2} \mathrm{~d} y+\frac{1}{2} g \eta^{2},
$$

where $g$ is the gravitational acceleration, which without any loss of generality is taken equal to 1 in the following.

Zakharov (1968) showed that the continuous Hamiltonian system defined by (1) with periodic boundary conditions can be transformed into an infinite dimensional discrete Hamiltonian system, which is more suitable for computational purposes. The perform this transformation we first introduce the following discrete Fourier transform of the two canonical variables $\eta(x, t)$ and $\psi(x, t)$ which are $2 \pi$ periodic functions of $x$

$$
\eta(x, t)=\sum_{-\infty}^{\infty} \eta_{k}(t) \mathrm{e}^{\mathrm{i} k x}, \quad \psi(x, t)=\sum_{-\infty}^{\infty} \psi_{k}(t) \mathrm{e}^{\mathrm{i} k x},
$$

where $\eta_{k}=\eta_{-k}^{*}$ and $\psi_{k}=\psi_{-k}^{*}$ because $\eta$ and $\psi$ are real. Now, consider the following linear transformation

$$
\left.\begin{array}{l}
\eta_{k}(t)=\frac{1}{2 \pi^{\frac{1}{2}}} \frac{|k|^{\frac{1}{2}}}{\omega^{\frac{1}{2}}(k)}\left[a_{k}(t)+a_{-k}^{*}(t)\right], \\
\psi_{k}(t)=-\frac{\mathrm{i}}{2 \pi^{\frac{1}{2}}} \frac{\omega^{\frac{1}{2}}(k)}{|k|^{\frac{1}{2}}}\left[a_{k}(t)-a_{-k}^{*}(t)\right],
\end{array}\right\}
$$

where $\omega(k)$ is the linear dispersion relation for deep water waves, which is given by

$$
\omega=\sqrt{ }|k| .
$$

Using this transformation the system given by (1) becomes the single equation

$$
\frac{\mathrm{d} a_{k}}{\mathrm{~d} t}=-\mathrm{i} \frac{\partial \mathscr{H}}{\partial a_{k}^{*}} .
$$

Notice that now $\mathscr{H}=\mathscr{H}\left(\boldsymbol{a}, \boldsymbol{a}^{*}\right)$ where $a=\left\{a_{k}\right\}$, and the functional derivatives have been replaced by partial derivatives. The function $\mathscr{H}\left(\boldsymbol{a}, \boldsymbol{a}^{*}\right)$ has the following invariances. $\mathscr{H}$ is invariant under the change $a \rightarrow a^{*}$ because of the reality of the energy. Also, because of the time reversibility of the system, $\mathscr{H}$ is invariant to the 


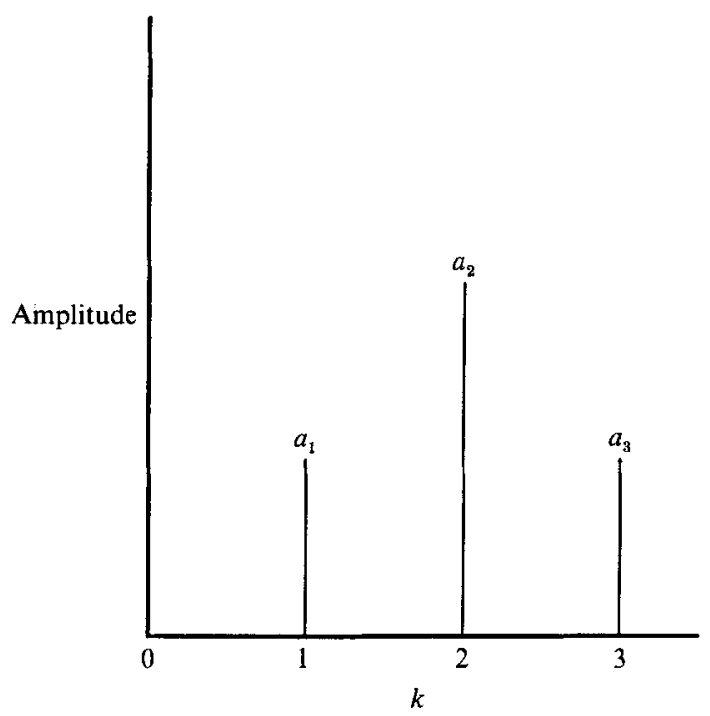

FIGURE 1. Spatial spectrum of the considered water wave.

change $a_{k} \rightarrow a_{-k}$. Further, the invariance of the system under horizontal translation leads to the fact that $\mathscr{H}\left(\boldsymbol{a}, \boldsymbol{a}^{*}\right)=\mathscr{H}\left(\boldsymbol{a}^{\epsilon},\left(\boldsymbol{a}^{\epsilon}\right)^{*}\right)$ where $\boldsymbol{a}^{\varepsilon}=\left\{a_{k} \mathrm{e}^{\mathrm{i} k \epsilon}\right\}$ for any $\epsilon$. The latter two invariances of the Hamiltonian $\mathscr{H}$ corresponds to two actual symmetries of the dynamical system, and therefore they lead to two corresponding integral quantities which are conserved during the motion (Benjamin 1984). These conserved quantities are the total energy $\mathscr{H}$ and the horizontal momentum $I$ which is defined as follows

$$
I=\sum_{-\infty}^{\infty} k a_{k} a_{k}^{*}
$$

The water wave system has another symmetry associated with the conservation of the total mass of the system. In (7) this conserved quantity appears in the fact that $a_{0}=0$.

Notice that to make use of the set of differential equations defined by (7) we need to evaluate the function $\mathscr{H}\left(a, a^{*}\right)$. In contrast to the momentum $I, \mathscr{H}$ cannot be expressed in terms of the coefficients $a$ in a simple way. However, it is simple to obtain a Taylor expansion of $\mathscr{H}$ in powers of the coefficients $\boldsymbol{a}$. We have performed this expansion up to fourth order in powers of the $a_{k}$. For a detailed description of how to perform the expansion see Zufiria (1987b).

In the present study we focus our attention on the case in which only three modes are allowed in the motion, i.e. we truncate the system (7) considering only three modes $a_{1}, a_{2}$ and $a_{3}$, and we take the rest of the modes identically equal to zero. This is the simplest case which leads to interesting dynamical behaviour. Notice that if less than three modes are considered the system is always integrable because the system possesses two integrals of the motion, $\mathscr{H}$ and $I$. With the scaling that we have assumed, the three wavenumbers that we allow are $k_{1}=1, k_{2}=2$ and $k_{3}=3$. They represent three free waves which travel to the right, i.e. $k$ positive. The spatial spectrum of our solutions will be limited to three modes, but on the other hand, we will keep the total resolution of the time evolution (figure 1).

The Hamiltonian energy that we obtain for the three-mode case is the following

$$
\mathscr{H}=H_{2}+H_{3}+H_{4}+O\left(a^{5}\right)
$$


where

$$
\left.\begin{array}{rl}
H_{2}= & a_{1} a_{1}^{*}+\sqrt{ } 2 a_{2} a_{2}^{*}+\sqrt{ } 3 a_{3} a_{3}^{*}, \\
H_{3}= & \frac{1}{2 \pi^{\frac{1}{2}}}\left[2^{\frac{3}{4}} 3^{\frac{1}{4}} a_{1} a_{2} a_{3}^{*}+2^{-\frac{3}{4}} a_{1}^{2} a_{2^{*}}\right]+\text { c.c., } \\
H_{4}= & \frac{1}{8 \pi}\left\{a_{1} a_{1}^{*}\left[a_{1} a_{1}^{*}+4 \frac{2 \sqrt{ } 2+1}{\sqrt{ } 2} a_{2} a_{2}^{*}+4(\sqrt{ } 3+3) a_{3} a_{3}^{*}\right]\right. \\
& +a_{2} a_{2}^{*}\left[8 a_{2} a_{2}^{*}+8 \frac{\sqrt{ } 3+6 \sqrt{ } 2}{\sqrt{ } 2} a_{3} a_{3}^{*}\right] \\
& \left.+27\left(a_{3} a_{3}^{*}\right)^{2}+\frac{6+4 \sqrt{ } 3+4 \sqrt{ } 6}{3^{\frac{3}{4}} \sqrt{ } 2}\left(a_{1} a_{3} a_{2}^{* 2}+\text { c.c. }\right)-3^{\frac{1}{4}}\left(a_{1}^{3} a_{3}^{*}+\text { c.c. }\right)\right\} .
\end{array}\right\}
$$

where c.c. stands for complex conjugate.

With this Hamiltonian and using (7) we can derive three complex ordinary differential equations to describe the time evolution of the three modes $a_{1}, a_{2}$ and $a_{3}$.

At this point we would like to comment on the comparison of our model with other sets of equations which have been used to describe the time evolution of a discrete spectrum of waves. In particular we focus our attention on one of the systems which has been commonly used in the past which is the discrete version. of Zakharov's integrodifferential equation (Yuen \& Lake 1982; Caponi et al. 1982; Yuen 1984). The integrodifferential Zakharov equation is a weakly nonlinear model which describes the slow time evolution of the dominant components of a wavetrain. The approximation, like ours, includes up to third-order terms in the amplitude of the waves. When a discrete spectrum with only one dominant component is considered, the assumption about a slow time evolution is equivalent to saying that the spectrum of the wave is composed of a dominant carrier $\left(k_{0}\right)$, and several small side bands $\left(k_{0}+\Delta k\right)$ with wavenumber close to the carrier, i.e. $\Delta k \ll 1$. When $\Delta k \approx a k_{0}^{2}$ this slow timescale is of order $a^{2} k_{0}^{2} \omega\left(k_{0}\right)$, where $\omega\left(k_{0}\right)$ is the linear dispersion relation for deep gravity waves, i.e. $\omega(k)=(|k|)^{\frac{1}{2}}$. In this case, three-wave interactions are the most important ones and they occur in the slow timescale. If $\Delta k \ll 1$ then two-wave interactions appear in the equations as higher than third-order effects, and therefore are not accounted for by the Zakharov model. When the narrow-band assumption is dropped two-wave interactions appear in the expansions. In particular when $\Delta k=$ 1 , they appear as second-order terms in the time evolution ODEs, i.e. $O\left(a^{2}\right)$. Hence, our model has quadratic terms in the ODEs which represent two-wave interactions and come from the third-order terms of the Hamiltonian, i.e. $H_{3}$.

The Zakharov system is not conservative (although it is phase-space volume preserving), and hence is not Hamiltonian. However, the discrete Zakharov equations happen to have more symmetries than the original water wave system. One consequence of this fact is that a three-mode model of the Zakharov equations can be integrated in closed form due to these additional symmetries when it should not be integrable, as follows from counting the symmetries of the real water wave problem. Because of this integrability we find that only periodic solutions for the coefficients are possible for three modes with the Zakharov approach, i.e. $a_{1}, a_{2}$ and $a_{3}$ are time periodic functions, which represents a modulation in the original wavetrain. A different approach to the three-wave problem based on the nonlinear Schrödinger equation, which describes the evolution of the wave envelope under the additional assumption of a narrow-band spatial process, followed by Stiassnie \& Kroszynski (1982) shows that in a three-wave model there exists another additional 
timescale which leads to a modulation-demodulation of the modulation recurrence phenomenon which is not present in the three-wave Zakharov model. Below we will show how our model contains this additional timescale and we will give the natural Hamiltonian explanation of it.

The presence of the two-wave interactions makes our model have fewer symmetries than the discrete Zakharov equations. Hence, the system is not integrable as we expected from the symmetries of the water wave problem.

\section{Steady waves and order reduction}

We start our analysis by looking at the steady solutions of our system. It is well known that the water wave equations admit permanent-form travelling-wave solutions which are of the form $a(t)=\left\{A_{k} \mathrm{e}^{\mathrm{i} k c t}\right\}$, where the $A_{k}$ are constants. To analyse these solutions it is convenient to write the equations in a frame of reference moving with speed $c$. In such a frame (7) becomes

$$
\frac{\mathrm{d} a_{k}}{\mathrm{~d} t}=-\mathrm{i} \frac{\partial \mathscr{H}}{\partial a_{k}^{*}}-\mathrm{i} c k a_{k}
$$

Notice that the system in this new frame of reference is also Hamiltonian, with new Hamiltonian $\mathscr{H}^{\prime}=\mathscr{H}+c I$. Now, for a given value of $c$ the equilibrium solutions of (11) correspond to permanent form travelling water waves which travel with a phase speed $c$.

For our three-mode model defined by the Hamiltonian given in (10), we have found that, besides the trivial solution $a_{1}=a_{2}=a_{3}=0$ for any value of $c$, which corresponds to a flat surface, the system has three different families of solutions with $c$ as parameter. These three families bifurcate from the flat surface and correspond to the so called class 1 , class 2 and class 3 waves. For the full water wave equations the three families correspond to the same wave but with a different scaling. A class $N$ wave has $N$ crests within the interval $(0,2 \pi)$. In the following we will call these families C1, C2 and C3 respectively. Even though they are the same wave with different scaling, once the scale of the problem is fixed the different families are different waves and play an important role in the dynamics of the system.

In our model, the C2 and C3 waves are identically the same wave with a different scaling, but the $\mathrm{C} 1$ is not identical to the others because of the truncation of the Hamiltonian. The $\mathrm{C} 2$ and $\mathrm{C} 3$ families are given exactly by

$$
a_{N} a_{N}^{*}=\frac{4 \pi}{N^{2}}\left(c-\frac{1}{N^{\frac{1}{2}}}\right), \quad a_{i}=0 \quad \text { for } i \neq N
$$

for $N=2,3$. For the $\mathrm{C} 1$ family we have the same expression as a first approximation, but there is a second-order correction. In figure 2 we show the three families of permanent-form travelling waves that our system contains.

The following step is to analyse the stability of the waves, which is equivalent to studying the stability of the fixed points of the Hamiltonian system defined by (11). The linear stability analysis will give us information about the local structure of the phase flow around the steady states. The stability eigenvalues are given by the eigenvalues of the following matrix (Saffman 1985; Zufiria \& Saffman 1986)

$$
S=\left(\begin{array}{cc}
M & N \\
-N^{*} & -M
\end{array}\right)
$$

where $\boldsymbol{M}=\partial^{2} \mathscr{H} / \partial a \partial a^{*}-c k l$ and $\boldsymbol{N}=\partial^{2} \mathscr{H} / \partial a^{* 2} . \boldsymbol{I}$ is the unit matrix. 


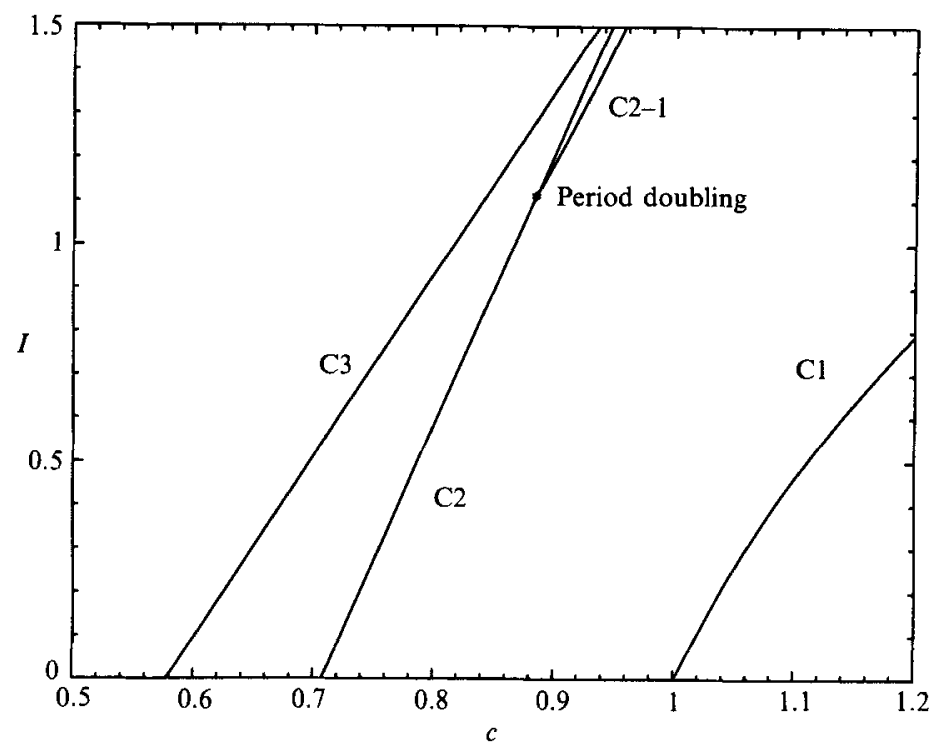

Frgure 2. Families of permanent-form travelling waves.

Some important properties of the matrix $S$ called 'Hamiltonian' (Arnold 1978) are that if $\sigma$ is an eigenvalue of $\boldsymbol{S}$, so is $-\sigma$, and so are the complex conjugates $\sigma^{*}$ and $-\sigma^{*}$. The matrix $S$ has always one eigenvalue that is zero due to the Galilean invariance. Because the system is even dimensional $\sigma=0$ has to be at least a double eigenvalue.

When the eigenvalues are pure imaginary the system is said to be neutrally stable. For $\sigma$ non-zero and pure imaginary the second derivative of the Hamiltonian is a non-degenerate quadratic form on the invariant space associated to the pair of eigenvalues $\sigma$ and $-\sigma$. If the eigenvalues are simple the quadratic form is either positive definite or negative definite. This is called the signature of the eigenvalue. The signature of an eigenvalue is preserved as the parameters are changed as long as the eigenvalue does not collide with another one. Stability is lost when some of the eigenvalues leave the imaginary axis. This can occur only by collision of two eigenvalues of different signature (MacKay 1986a; MacKay \& Saffman 1986).

We have computed the eigenvalues along the three families of waves, and the results are shown in figures $3(a-c)$. We can observe that from the dynamical point of view the family $\mathrm{C} 2$ seems to be the most interesting. In this family we have several regions where the stability of the wave changes. Within the range of our computations we can also see that for the $\mathrm{C} 3$ wave family there is no loss of stability. There is a crossing of two eigenvalues, but the crossing does not lead to a change of stability because the eigenvalues have the same signature. Longuet-Higgins (1978) performed the computations for the full water wave equations. Looking at his and our results, we can observe that our eigenvalues have an identical qualitative behaviour to the ones computed by Longuet-Higgins.

We have an instability region due to the collision of the eigenvalues $(3,2)$ and $(1,2)$. The notation $(n, m)$ refers to the eigenvalue whose eigenvector represents a perturbation of $n$ crests over a wave of $m$ crests in the $(0,2 \pi)$ interval, see LonguetHiggins (1978) and Zufiria $(1987 b)$. This kind of instability has been extensively studied in the literature (Benjamin \& Feir 1967; Longuet-Higgins 1978). It corresponds to a modulation of the $\mathrm{C} 2$ wave by small side bands. But most of the 

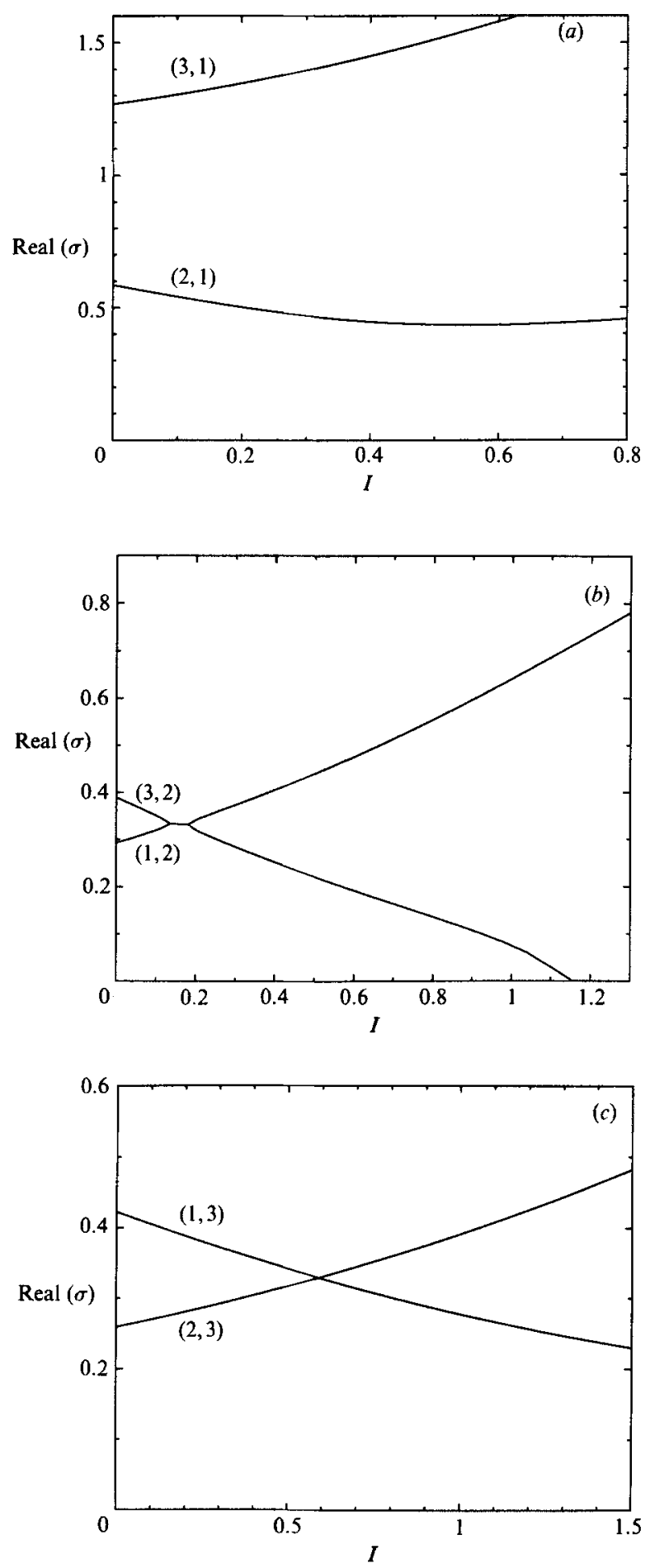

Figure 3. Linear stability eigenvalues for the families of permanent-form travelling waves: (a) C1 branch. (b) C2 branch. (c) C3 branch. 
(a)

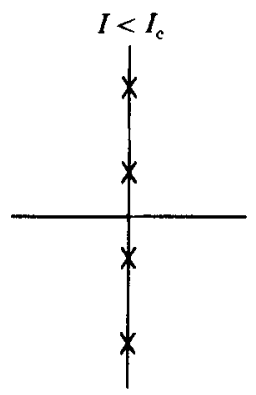

(b)

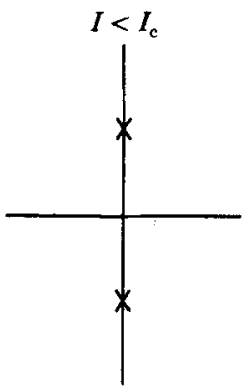

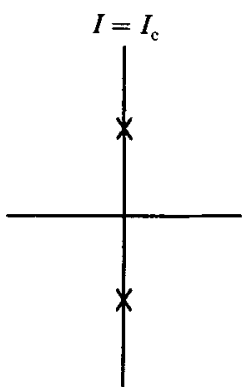

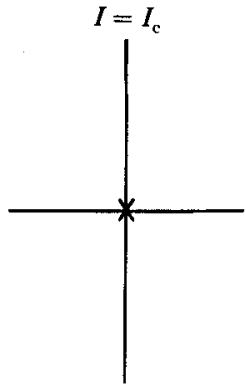

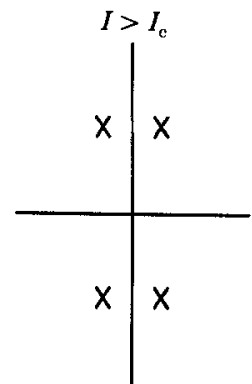

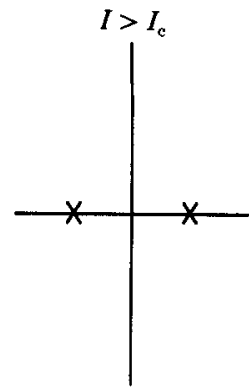

Figure 4. Behaviour of the stability eigenvalues on the $\sigma$ plane: (a) Hamiltonian Hopf bifurcation. (b) Period doubling bifurcation. In both figures $I_{\mathrm{c}}$ represents the corresponding bifurcation value.

previous study has been limited to the case in which the frequencies of the side bands are very close to the carrier. For a given frequency of the side bands, i.e. given $\Delta k$, there exists an amplitude at which the wave becomes unstable. When $\Delta k \ll 1$, the instability appears at very low amplitude waves and is called the Benjamin \& Feir instability. As the frequency of the side bands approaches that of the carrier, the amplitude at which the instability appears goes to zero. In the present case the carrier is the $\mathrm{C} 2$ wave with wavenumber $k=2$ and the side bands have wavenumbers $k=1$ and $k=3$. We will see below that there is no qualitative change when $\Delta k$ is varied. The only changes appear in the amplitude of the waves and in the timescale in which the perturbations evolve.

In the Hamiltonian literature this change of stability is known as the HamiltonianHopf bifurcation (Van der Meer 1985). In this bifurcation, four pure imaginary eigenvalues collide in pairs on the imaginary axis and split into two complex conjugate pairs of eigenvalues leaving the imaginary axis (see figure $4 a$ ). This bifurcation has been studied very deeply in Celestial Mechanics because it appears in the stability of one equilibrium point of the planar three-body problem (Jacobi 1836; Routh 1875; Poincare 1890; Brown 1911 ; Buchanan 1941). But the bifurcations of periodic orbits around a bifurcation of this kind were not completely understood until the recent work of Van der Meer $(1985,1986)$. In the following section we will perform the analysis numerically for our model and we will see that our results agree with those of Van der Meer.

Besides this instability, we do find another instability in the C2 family. We observe that as the phase speed of the wave is increased one of the eigenvalues decreases and becomes zero at one point. At this point the eigenvalue $\sigma$ collides with its symmetric $-\sigma$ and leaves the imaginary axis, becoming real (see figure $4 b$ ). As the eigenvalues go through zero there must be a bifurcation point. This bifurcation corresponds to 
the period-doubling bifurcation which was first found by Chen \& Saffman (1980) for the full water wave equation. Further studies of bifurcations have been done by Saffman (1980) and Longuet-Higgins (1985, 1986). At this period-doubling bifurcation the $\mathrm{C} 2$ wave becomes unstable and a new family of permanent form travelling waves is created. Along the new family the waves have two crests in the interval $(0,2 \pi)$, but now the two crests have different heights. Notice that the period of this wave is $2 \pi$, whereas the period of a $\mathrm{C} 2$ wave is $\pi$, therefore the bifurcation is called period-doubling bifurcation. This new branch is shown in figure 2 .

We have also computed the eigenvalues along the new branch. They are pure imaginary within the range of our computations. Hence, the new branch is stable.

Now that we have a picture of the fixed points of our system and their stability, we can go into the study of more complex elements of the dynamics. As we have already mentioned, the water wave system has two time-independent integrals of the motion which are the energy $\mathscr{H}$ and the horizontal momentum $I$. With the use of these symmetries the order of the system can be reduced. In particular, when a timeindependent integral of the motion is known, there exists a reduced Hamiltonian system including two fewer variables with the property that every solution of the original system can be determined using a quadrature from those of the reduced system (Olver 1986, p. 402).

In our particular case we have a three-degrees-of-freedom Hamiltonian system and using the horizontal momentum we will be able to reduce our system to a twodegrees-of-freedom Hamiltonian system. The general methodology to perform the transformation is based on taking the conserved quantity as one of the new variables. As we are going to be mainly interested in perturbations of $\mathrm{C} 2$ waves, we will remove the variable $a_{2}$ from the system. The transformation that we introduce is the following one

$$
a_{1}=\hat{a}_{1} \mathrm{e}^{\mathrm{i} \theta}, \quad a_{2}=\left|a_{2}\right| \mathrm{e}^{2 \mathrm{i} \theta}, \quad a_{3}=\hat{a}_{3} \mathrm{e}^{\mathrm{i} \theta},
$$

where

$$
\left|a_{2}\right|^{2}=\frac{1}{2}\left(I-\hat{a}_{1} \hat{a}_{1}^{*}-3 \hat{a}_{3} \hat{a}_{3}^{*}\right) .
$$

Notice that before we had $\mathscr{H}=\mathscr{H}\left(a_{1}, a_{2}, a_{3}, a_{1}^{*}, a_{2}^{*}, a_{3}^{*}\right)$ and now we have $\mathscr{H}=$ $\mathscr{H}\left(I, \theta, \hat{a}_{1}, \hat{a}_{3}, \hat{a}_{1}^{*}, \hat{a}_{3}^{*}\right)$. The new Hamiltonian that we obtain after the transformation is

$$
\mathscr{H}=E+\frac{1}{2} \omega_{2} I+\frac{1}{4} \omega_{22} I^{2},
$$

where

and

$$
\begin{aligned}
E= & W_{1} \hat{a}_{1} \hat{a}_{1}^{*}+W_{3} \hat{a}_{3} \hat{a}_{3}^{*}+W_{11} \hat{a}_{1}^{2} \hat{a}_{1}^{* 2}+W_{13} \hat{a}_{1} \hat{a}_{1}^{*} \hat{a}_{3} \hat{a}_{3}^{*}+W_{33} \hat{a}_{3}^{2} \hat{a}_{3}^{* 2} \\
& +\left[\frac{1}{2}\left(I-\hat{a}_{1} \hat{a}_{1}^{*}-3 \hat{a}_{3} \hat{a}_{3}^{*}\right)\right]^{\frac{1}{2}}\left[w_{123}\left(\hat{a}_{1} \hat{a}_{3}^{*}+\hat{a}_{1}^{*} \hat{a}_{3}\right)+w_{112}\left(\hat{a}_{1}^{2}+\hat{a}_{1}^{* 2}\right)\right] \\
& +\frac{1}{2} w_{1322}\left[I-\hat{a}_{1} \hat{a}_{1}^{*}-3 \hat{a}_{3} \hat{a}_{3}^{*}\right]\left[\hat{a}_{1} \hat{a}_{3}+\hat{a}_{1}^{*} \hat{a}_{3}^{*}\right]+w_{1113}\left(\hat{a}_{1}^{3} \hat{a}_{3}^{*}+\hat{a}_{1}^{* 3} \hat{a}_{3}\right),
\end{aligned}
$$

The coefficients $w$ are the ones corresponding to the Hamiltonian of (10), e.g. $w_{1322}$ is the coefficient of the term $a_{1} a_{3} a_{2}^{*} a_{2}^{*}$.

Notice that $I$ and $\theta$ are action and angle variables, and therefore the new Hamiltonian does not depend on $\theta$ and the equations for the two variables $I$ and $\theta$ 
are uncoupled from the rest of the equations. So our system has been reduced to a two-degree-of-freedom Hamiltonian system with the variables $\hat{a}_{1}=p_{1}+\mathrm{i} q_{1}$ and $\hat{a}_{3}=$ $p_{3}+\mathrm{i} q_{3}$, and the momentum $I$ acts as a parameter of the system. To go back to the original variables we need $\theta$ which is given by

$$
\Omega=\frac{\mathrm{d} \theta}{\mathrm{d} t}=-\frac{\partial \mathscr{H}}{\partial I}
$$

For the reduced Hamiltonian system of two degrees of freedom either $E$ or $\mathscr{H}$ can be considered as Hamiltonians. The difference between $\mathscr{H}$ and $E$ is just the choice of the zero energy level. $\mathscr{H}$ measures the absolute energy whereas $E$ measures the energy with respect to the $\mathrm{C} 2$ wave. When $E=0$ then $\mathscr{H}$ is the energy of the C2 wave of momentum $I$.

For the Hamiltonian (17) the origin is a fixed point for all values of $I$. This fixed point corresponds to the $\mathrm{C} 2$ wave of momentum $I$.

Our problem has been reduced to the study of the properties of a two-degrees-offreedom Hamiltonian system, i.e. a set of four first-order ordinary differential equations for the four real variables $p_{1}, q_{1}, p_{3}$ and $q_{3}$. This set of equations come from applying (1) to the reduced Hamiltonian given by (17).

\section{Two-frequency solutions}

In the previous chapter we studied the steady states of our system. These solutions correspond to permanent form travelling waves as we have already mentioned. These solutions are very well known and have been a major subject of study in the field of water waves. They are the first step in the understanding of the dynamics of water waves. We have found that our model describes qualitatively these solutions and their stability. Now we go one step further and we study the second important elements in the motion of any dynamical system. These elements are the periodic orbits. We mean by periodic orbits solutions in which $\hat{a}_{1}$ and $\hat{a}_{3}$ are periodic functions of the time.

Physically these solutions represent waves with two frequencies, i.e. periodic modulations to travelling waves. The two basic frequencies are the frequency associated with the basic carrier wave $\Omega$ and the modulation frequency $2 \pi / T$ where $T$ is the period of $\hat{a}_{1}$ and $\hat{a}_{3}$.

We show that these periodic solutions exist in the water wave problem and they are crucial elements in understanding the dynamics of the water wave system.

Let us concentrate our attention on a $\mathrm{C} 2$ wave. Starting from zero amplitude (i.e. $I=0$ ) and going along the $\mathrm{C} 2$ family (see figure 2 ) we know that the steady wave is neutrally stable for small values of the momentum. This is because the four stability eigenvalues (see figure $3 b$ ) are pure imaginary. They are of the form $\pm i \omega_{1}$ and $\pm i \omega_{3}$.

According to the Liapunov theorem for fixed points of Hamiltonian systems (Abraham \& Marsden 1978), for a given fixed value of $I$ the four-dimensional phase space of our system has two two-dimensional manifolds containing the $\mathrm{C} 2$ wave, which is the origin of our system. Each of these two-dimensional manifolds contains a one-parameter family of periodic orbits whose periods approach $2 \pi / \omega_{1}$ or $2 \pi / \omega_{3}$ respectively as the amplitude goes to zero and the orbit approaches the fixed point. This is actually true when there is no resonance relation between the two frequencies $\omega_{1}$ and $\omega_{3}$. We say that two frequencies are resonant when there exist two integers $n$ and $m$ such that $\omega_{1} m+\omega_{3} n=0$. In resonant cases the linear stability analysis is 
not sufficient to determine the local structure of the phase flow around the fixed points.

The fact that periodic orbits appear as one parameter families is a degeneracy of Hamiltonian systems. In our model these families of periodic orbits will depend on two parameters which are $I$ and $T$. By changing $I$ we change our system, and therefore the phase space. On the other hand, for a given $I$ changing $T$ we go from one periodic orbit to another but without changing the phase space. As the system is Hamiltonian the energy is conserved during the time evolution, and hence each periodic orbit lies in a constant energy $E$ surface. In a three-dimensional space with $T, I$ and $E$ as coordinates, the families of periodic orbits form a two-dimensional surface. Our first step is to analyse the structure of this surface as well as the stability of the periodic solutions, i.e. two-frequency waves.

We start by considering a given fixed value of the momentum $I$ and we go along the families continuing with the period $T$. The numerical computations for these orbits of our four-dimensional dynamical system were performed using the code AUTO developed by Doedel \& Kernevez (1986). This code allows one to perform continuations of periodic orbits of general dynamical systems by writing the problem as a boundary-value problem. For more details about this code see Doedel \& Kernevez (1986). This code has already been successfully used by Zufiria $(1987 a, c)$ to study the bifurcation structure of periodic gravity and gravity-capillary permanent-form travelling waves.

In our computations we took $I=0.05$. For this value of the momentum we found that the family of periodic orbits starting with period $T=2 \pi / \omega_{1}$ at $\mathrm{C} 2$ ends in a periodic solution which corresponds exactly to a $\mathrm{C} 1$ wave. Notice that with the transformation introduced in (14), a permanent form $\mathrm{C} 1$ is a periodic solution with period $T$ defined as follows

$$
\frac{2 \pi}{T}=\Omega-c,
$$

where $c$ is the phase speed of the $\mathrm{C} 1$ wave. Along the branch we find that the solutions correspond to sinusoidal oscillations of the four variables $p_{1}, p_{2}, q_{1}$ and $q_{3}$. As we approach the end point of the branch the amplitudes of the oscillations of $p_{3}$ and $q_{3}$ decay to zero and we end up with an orbit which is contained in the $\left(p_{1}, q_{1}\right)$ plane of the four-dimensional phase space. In the following we will refer to this family of periodic orbits as the $\mathrm{P} 21$ family because it connects the $\mathrm{C} 2$ waves with the $\mathrm{C} 1$ waves. So these families of two-frequency waves are natural ways of connecting steady waves of different classes. In some sense these waves can be interpreted as the nonlinear superposition of two waves of different wavelength.

We repeated the same calculations along the other branch. In this case we found that the family ends again in a wave which corresponds to a steady C3 wave. This family will be named P23.

In figure $5(a)$ we show the projection of several members of the P21 family over the $\left(p_{1}, q_{1}\right)$-plane. The central point corresponds to the $\mathrm{C} 2$ wave and the outer periodic orbit to the $\mathrm{C} 1$ wave. In figure $5(b)$ we do the same for the P23 family. Along these families as we go from one orbit to the other the energy of the orbits is changing. For the P21 family orbits the energy is positive and increases monotonically, as well as the period $T$. For the P23 family the energy and the period decrease monotonically. Figure $5(c)$ shows the two branches of solutions.

In order to understand the physical meaning of these kinds of solutions we have plotted in figures $5(d)$ and $5(e)$ the elevation of the physical surface of the wave $\eta(x, t)$ at $x=0$ versus time for different members of the families. We can see how 

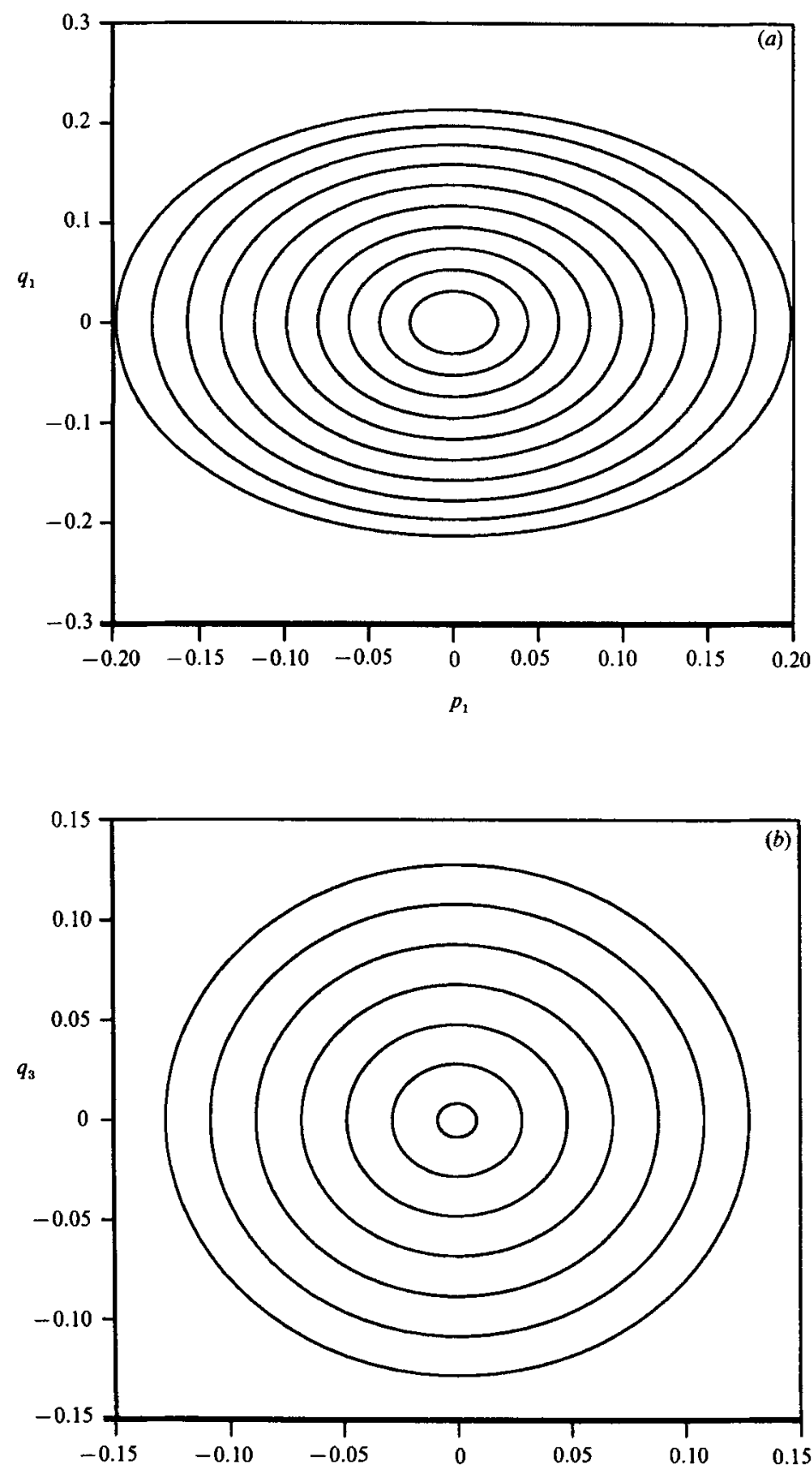

$p_{3}$

Figure 5. Families of periodic orbits for $I=0.05$. (a) Projection onto the $\left(p_{1}, q_{1}\right)$ plane of some members of the P21 family of periodic orbits. (b) Projection onto the $\left(p_{3}, q_{3}\right)$ plane of some members of the P3 family. (c) Families of periodic orbits. $(d)$ Elevation of the surface $\eta(0, t)$ for some members of the P21 family. (e) Elevation of the surface $\eta(0, t)$ for some members of the P23 family. 

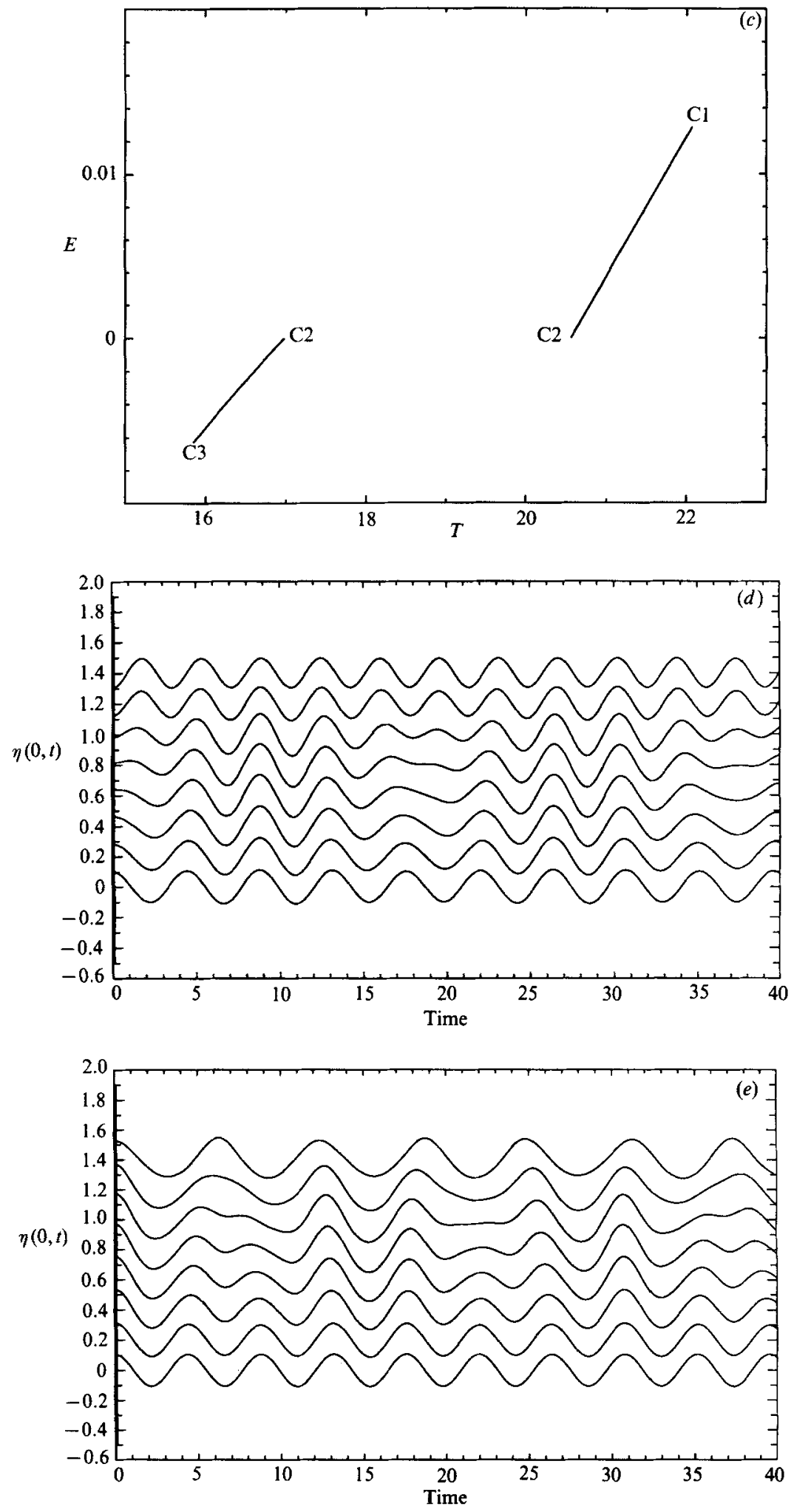

Figure 5(c-e). For caption see page 353. 
starting from a C2 wave we approach gradually the C1 and C3 families by going along the branches of solutions, i.e. changing the energy of the solutions.

The stability of these periodic solutions is determined by the Floquet multipliers of the orbits. The Floquet multipliers are a measure of the growth of linear perturbations of the orbits in one period $T$. In our four-dimensional model for each periodic orbit we have four Floquet multipliers. When at least one of the multipliers of the orbit is larger than 1 in absolute value the periodic orbit is said to be unstable. When all the multipliers are inside the unit circle the system is stable and the periodic orbit is attracting. Any initial perturbation will die and the system will end in the periodic solution. In the case of Hamiltonian systems, because the flow preserves volume in the phase space the product of all the Floquet multipliers has to be equal to one. A periodic orbit always has a multiplier which is equal to +1 corresponding to sliding a little along the orbit. For Hamiltonian systems as the evolution is on constant energy surfaces, there is another multiplier equal to +1 . Finally from the reality of the Hamiltonian, it follows that the two other multipliers will be conjugate points $\sigma, \sigma^{*}$ on the unit circle or reciprocal points $\sigma, 1 / \sigma$ on the real axis (Green et al. 1981). In the following section we will see the importance of the stability of the periodic orbits on the structure of the phase space.

Besides performing the continuation of the periodic orbit, the code AUTO also provides the Floquet multipliers of the orbits. We have found for the families P21 and P23 the multipliers lie on the unit circle along the whole branch. This means that these periodic orbits are stable.

As we increase the momentum $I$, the structure remains the same up to the point at which the C2 family loses stability. This happens for a value of the momentum of $I \approx 0.135$. This point corresponds to the so called Benjamin \& Feir instability, also called class 1 instabilities. For $I$ larger than this value the eigenvalues for the linear stability of the C2 wave are not pure imaginary any more. Hence the Liapunov theorem cannot be applied and it is expected that the families of periodic orbits will not contain the C2 point any more. At the critical point at which the instability happens we have $\omega_{1}=\omega_{3}$ so the two families start with the same period $T$ at $\mathrm{C} 2$. This means that the infinitesimal perturbation waves riding on the $\mathrm{C} 2$ all have the same period. In other words, the two families connect at this point. Above this point we have found that the two families become a single family which connects the C1 with the $\mathrm{C} 3$ without going through the $\mathrm{C} 2$ wave. We have performed the computations for $I=0.017$, and the energy of the periodic orbits along the branch is shown in figure 6 .

This change of structure of the families of periodic orbits as a Hamiltonian Hopf bifurcation is crossed was already expected from the results of Van der Meer (1985). He performed the unfolding of this bifurcation by using normal form theory for the energy-momentum maps. He found that the two-dimensional surface formed by the periodic orbits in a three-dimensional space with coordinates $I, E$ and some norm of the orbits has a swallowtail singularity. To show this form in our computations we define the following norm for our periodic orbits

$$
\|a\|^{2}=\frac{1}{T} \int_{0}^{T}\left(p_{1}^{2}+q_{1}^{2}+p_{3}^{2}+q_{3}^{2}\right) \mathrm{d} t .
$$

Using this norm in figure 7 we give a two-dimensional projection of this surface.

Again we have found that the family is stable along the whole branch, i.e. the Floquet multipliers lie on the unit circle. 


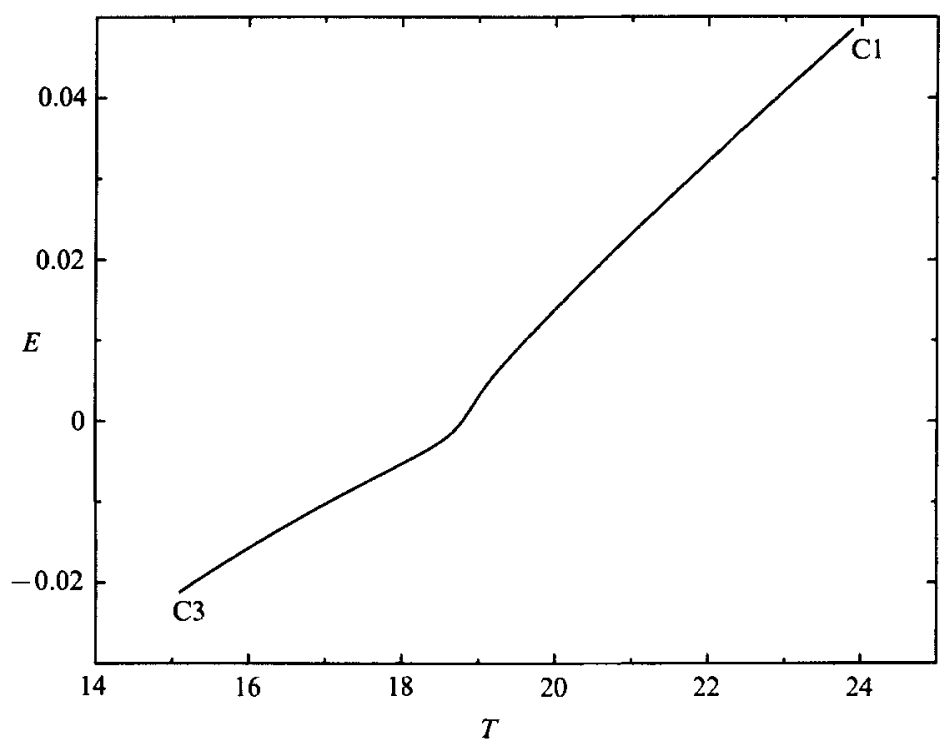

Figure 6. P13 family of periodic orbits for $I=0.17$.

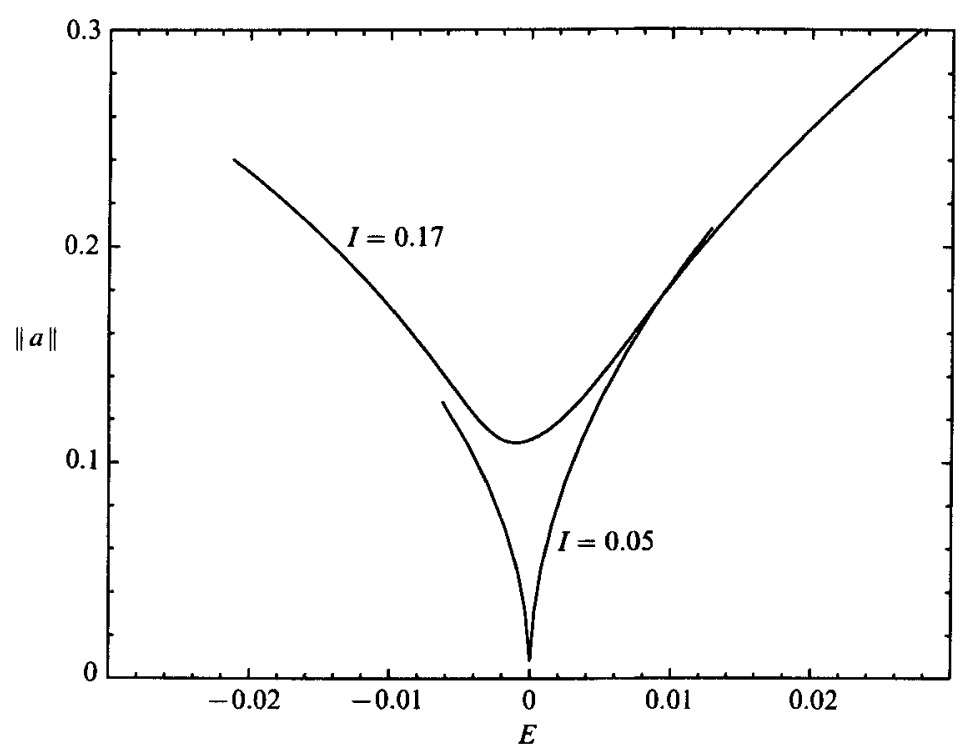

Figure 7. Projection onto the $(E,\|a\|)$-plane of the swallowtail singularity.

If we keep increasing the momentum along the $\mathrm{C} 2$ branch we find the steady solution becomes stable again (see figure $3 b$ ). At approximately $I \approx 0.18$ we have an inverse Hamiltonian Hopf bifurcation and the eigenvalues go back to the imaginary axis. Above this new critical value one can apply the Liapunov theorem again and it follows that there are two families of periodic orbits which contain the $\mathrm{C} 2$ wave. One may think that the P13 family connects again with the fixed point, i.e. C2, and splits into the two families P21 and P23, but this is not what happens. What really happens is that a new family of periodic orbits is created. This new family connects C2 with itself. This new family approaches the fixed point C2 with two different 


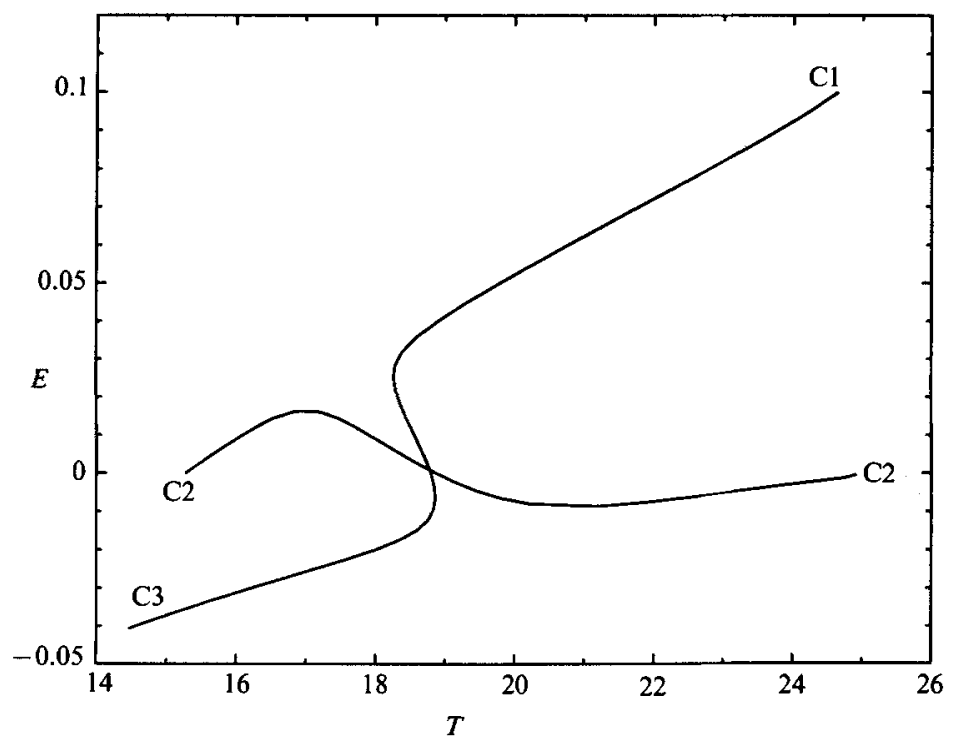

Figure 8. Families of periodic orbits $\mathrm{P} 13$ and $\mathrm{P} 22$ for $I=0.4$.

periods $2 \pi / \omega_{1}$ and $2 \pi / \omega_{3}$. The surface corresponding to this new family in the $(I, E$, $\|a\|)$ space has the form of a cone, with the vertex at $I \approx 0.18, E=0$ and $\|a\|=0$.

Besides this new branch of solutions that we will call P22, we still have the P13 family. The two families are shown in figure 8. One important characteristic of the new P22 family is that it is unstable in some finite interval along the branch. As we will see in the following sections this will give the system the possibility of undergoing very complex motions.

As we increase the momentum we find that $\omega_{1} \rightarrow 0$. This means that one of the limiting periods of the P22 family goes to infinity. This suggests the existence of heteroclinic or homoclinic connections as we approach the period-doubling bifurcation. These are orbits that go through fixed points and therefore they have infinite period.

According to Longuet-Higgins (1978) stability computations for the full water wave problem, the $I=0.135$ where the $C 2$ wave loses stability corresponds to a water wave of amplitude $a k=0.229$. The point where the $\mathrm{C} 2$ wave becomes stable again $(I=0.18)$ corresponds to $a k=0.367$. And finally, the amplitude for the perioddoubling bifurcation given by Chen \& Saffman (1980) is $a k=0.405$ which corresponds to the point $I=1.15$ of our model (see figure $3 b$ ). These numbers give an idea of the steepnesses for which the different families of two-frequency waves can be expected to occur in the case of real water waves.

\section{Quasi-periodic solutions and irregular motion}

For a dynamical system the fixed points and periodic solutions play the same role in the phase space as the bones in the human body. In some sense, these elements and their stability properties determine the basic structure of the phase space. But all this does not mean that these motions are the most probable motions of the system. Actually, in Hamiltonian systems the motions represented by the fixed points and periodic solutions occur for a set of initial conditions which are zero. This is due to the fact that in Hamiltonian systems these solutions cannot be attracting. 
However, when unstable fixed points or periodic orbits exist, the stable and unstable manifolds of these elements divide the phase space into invariant regions with different kinds of motions. In many cases these invariant manifolds are the cause of a very complex dynamics.

Typically in a Hamiltonian system the motion is neither entirely regular, nor entirely irregular, but the phase space is made up of a complicated mixture of regular and irregular regions (MacKay, Meiss \& Percival 1984). In the regular regions the motion is contained in invariant tori or 'KAM surfaces'. The irregular components of the motions have a stochastic or chaotic character which fills higher dimensional regions. When the system is integrable the motion is completely regular and there is no net transfer of energy among the different degrees of freedom of the system. There is mixing only in the phase of the oscillations (MacKay 1986b).

To continue our analysis we are going to reduce the study of our system to the study of the properties of a two-dimensional area-preserving map. To do this, consider a hypersurface $\Sigma$ of dimension one less than the phase space which is transverse to the flow (i.e. the flow is nowhere tangent to $\Sigma$ ). Such a surface is known as a surface of section (Poincaré 1885). Now let us consider the following map. We take one point on $\Sigma$ and integrate the orbit until the orbit crosses the surface again. The orbit may not come back to the surface in which case the map is not defined at that point. But for the orbits that hit the surface again we have a very well-defined map from $\Sigma$ into itself. Since in Hamiltonian systems the orbits lie on constant energy surfaces the hypersurface $\Sigma$ can be decomposed into a one-parameter family of hypersurfaces $\Sigma_{E}$ of dimension two less than the phase space with the energy $E$ as parameter. Each of these hypersurfaces $\Sigma_{E}$ is mapped into itself by the first return map. This map is frequently called the Poincare map.

Following this procedure we can reduce the study of our system to the study of an area-preserving map with two parameters, which are the energy level $E$ and the momentum $I$. In our problem the surface of section was chosen in the following way

$$
\Sigma_{E}=\left\{\left(p_{1}, q_{1}, p_{3}, q_{3}\right): q_{3}=0, \quad \mathrm{~d} q_{3} / \mathrm{d} t>0, \quad E=E\left(p_{1}, q_{1}, p_{3}, q_{3} ; I\right)\right\}
$$

For most of the pairs of values of $E$ and $I$, a point lying on this surface is determined by giving the set of coordinates $\left(p_{1}, q_{1}\right)$. For a pair $\left(p_{1}, q_{1}\right)$ and given values of $E$ and $I$, we can determine the value of $p_{3}$, and then $\left(p_{1}, q_{1}, p_{3}, 0\right)$ is a point on $\Sigma_{E}$. Hence in the following we will consider our Poincaré map as a map from the plane $\left(p_{1}, q_{1}\right)$ into itself. However, when the momentum $I$ is large the one-to-one correspondence between the points of the $\Sigma$ surface and the $\left(p_{1}, q_{1}\right)$-plane is lost and some non-uniqueness problems appear in the projection of the map.

We have to mention that the surface defined by (22) is transverse to the flow everywhere except at the origin of the phase space. At the origin the gradient of the energy is null and the flow is not transverse to any surface. As the origin is contained in the surface $\Sigma_{0}$, we will have to be careful when interpreting some results for the map at this surface level. We will deal with this surface level during most of the work because, as we will see below, this is the surface for which the most interesting results occur.

The periodic orbits that we studied in the previous section are fixed points of the map. Also, at the zero energy surface we have another degenerate fixed point of the map which is the origin of the phase space, i.e. the steady C2 wave. We say that this fixed point is degenerate in the sense that it is also a fixed point of the flow.

Let us start analysing the behaviour of our map as we change the parameters $E$ and $I$. Recall that as we change the energy level $E$ we do not change the phase space, 
but we are just looking at different slices of the same phase space. On the other hand, as we change the momentum $I$ we are changing the phase space. We started by taking $I=0.05$. For this value of the momentum we know from figure $5(c)$ that at each energy level we have only one fixed point. In particular for the surface $E=0$, we only have a fixed point which is the C2 wave (see figure $9 a$ ). By using an ODE solver we have integrated in time our four-dimensional system and computed the map. In figure $9(a)$ we have plotted 1000 iterations of the map for 16 initial conditions equally spaced along the $q_{1}=0$ axis. We can see that the motion is completely regular and all the orbits lie on invariant tori (invariant circles of the map). As the origin $\mathrm{C} 2$ is a marginally stable fixed point, the point appears as a centre on the map. For any other energy level we found the same structure for the map. Hence, we follow that for this value of the momentum the only possible motion for the system is quasi-periodic. Physically this means that the expected motion of a water wave with these parameters is the one of a carrier wave modulated with two frequencies. One of them is related to the return time of the map, and the other one is related to the rotation number of the map for the corresponding invariant circle (Guckenheimer \& Holmes 1983). From the regular structure of the map it is apparent that the map is integrable for these values of the parameters. In figure $9(b-d)$ we show the time evolution of the two side-band modes $a_{1}$ and $a_{3}$ and the spectrum of $\left|a_{1}(t)\right|$ for initial conditions $p_{1}=0.1, q_{1}=0$.

As we increase the value of $I$, the structure of the map does not change up to the point in which the $\mathrm{C} 2$ wave loses stability. As follows from figure 6 , in the unstable region for a general energy level the map has only one fixed point. The $\Sigma_{0}$ surface, however, has two fixed points, one of them corresponds to the zero energy periodic solution of the family $\mathrm{P} 13$ and the other one is the C2 wave. As the family of periodic orbits P13 is stable, the corresponding fixed point will be a centre. The other fixed point will be a saddle. In figure $10(a)$ we show the map for $I=0.17$ and $E=0$. Again we have plotted 1000 iterations of several orbits starting on the $q_{1}=0$ axis.

Only half of the stable and unstable manifolds of the origin appear in the plot. This is because we are plotting the intersections in which $\mathrm{d} q_{3} / \mathrm{d} t>0$ and the origin is a degenerate fixed point of the map in the sense that the flow is not transversal to the $\Sigma_{0}$ at this point. For half of the manifolds $\mathrm{d} q_{3} / \mathrm{d} t$ is positive and for the other half negative. As we go through the origin the sign changes.

In figure $10(a)$ we can see that the stable and unstable manifolds of the origin coincide. These manifolds are just a section of the manifolds of the original flow. According to the linear stability eigenvalues, the origin is a saddle-focus. Hence, the stable and unstable manifolds are bidimensional. As both of them have to be contained in the three-dimensional surface of zero energy, the two manifolds will generically intersect transversally within that surface. When this intersection is transverse, Devaney (1976) has proven that the motion is irregular within the region close to the manifolds. Our numerical results suggest that in our case the intersection of the manifolds is not transverse and they actually coincide. Lerman \& Umanskii (1984) have shown that for integrable Hamiltonian systems the two manifolds generically coincide. It seems that this is our case and the system is integrable for these values of the parameters.

For $I=0.17$ we find that for generic initial conditions the motion occurs again on invariant tori. Hence, we expect that for most of the initial conditions we will have a quasi-periodic motion, i.e. a wave with three frequencies, a carrier frequency, a modulation of the carrier, and a new modulation-demodulation period. Up to this point, nothing has changed from the previous case in which $I<0.135$. But there is 


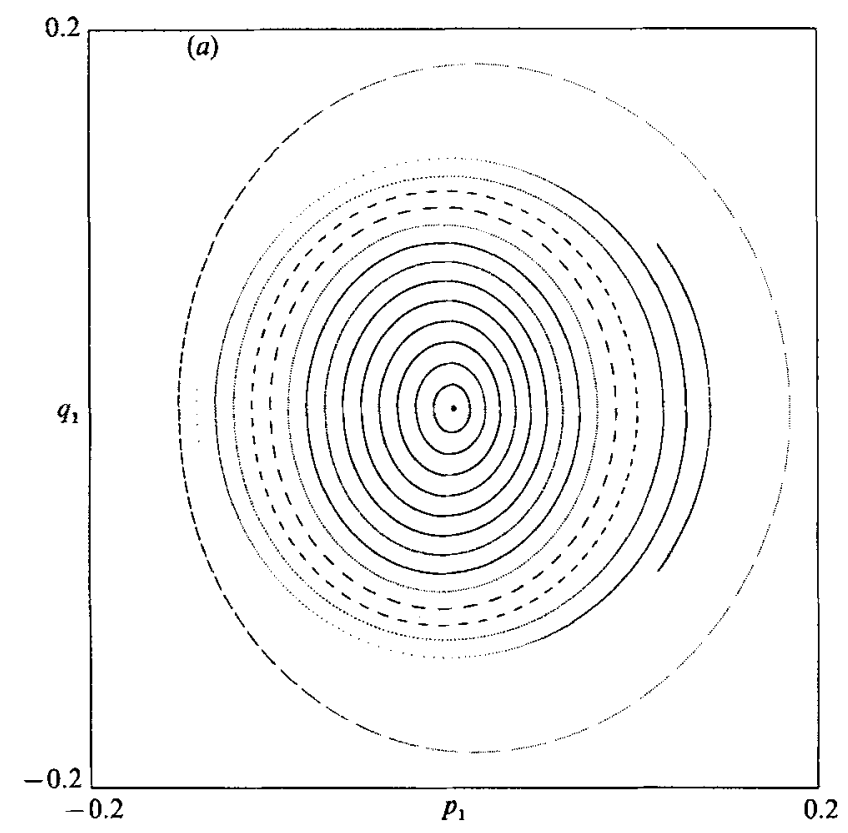

(b)

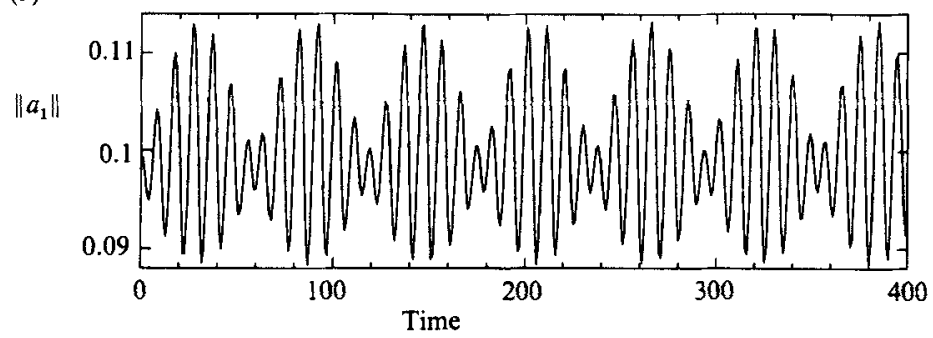

(c)

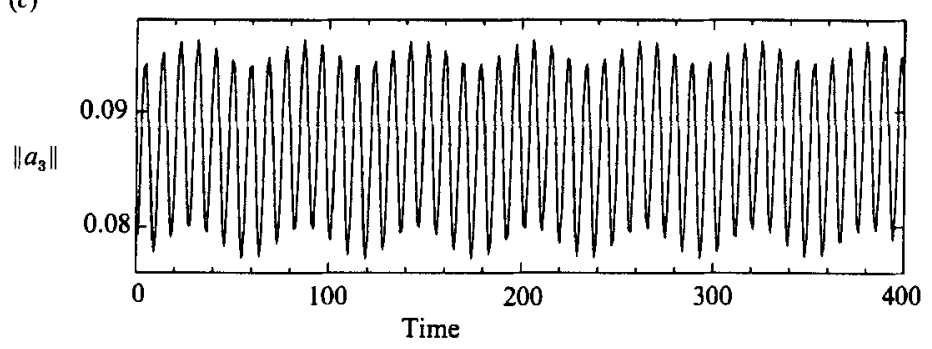

(d)

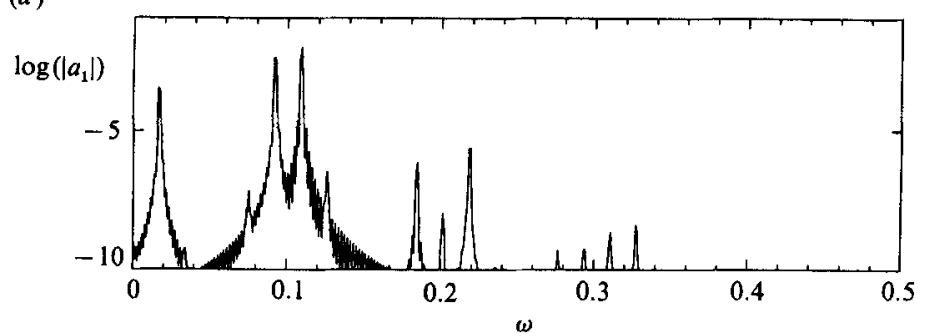

Figure 9. Results for $I=0.05$. (a) Poincaré map for the $E=0$ surface. (b) Time evolution of modulus of $a_{1}$ for initial conditions $p_{1}=0.1$ and $q_{1}=0$. (c) Time evolution of modulus of $a_{3}$ for the same initial conditions. $(d)$ Temporal spectrum of modulus of $a_{1}$ for the same initial conditions. 

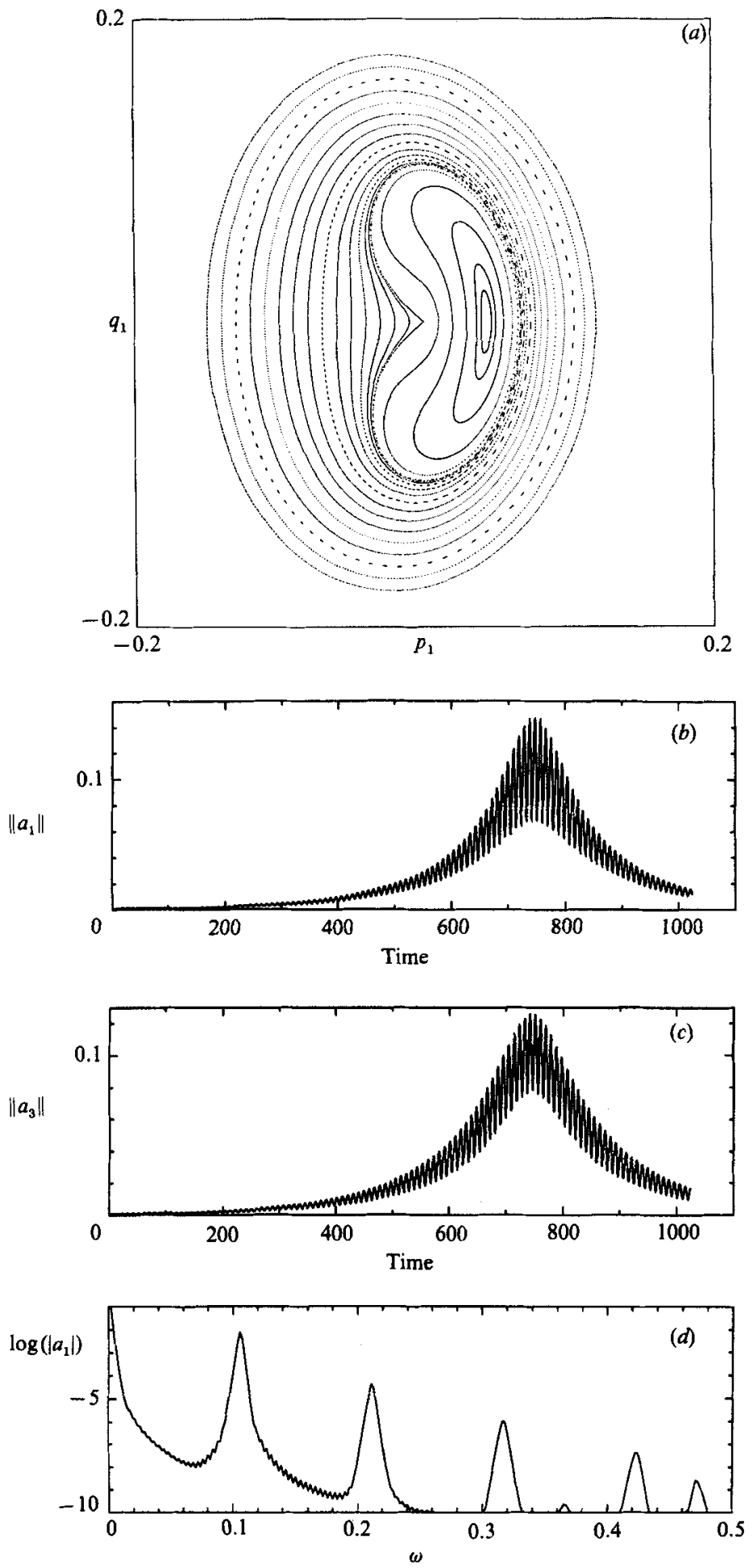

Figure 10. Results for $I=0.17$. (a) Poincare map for the $E=0$ surface. (b) Time evolution of modulus of $a_{1}$ for initial conditions $p_{1}=0.001$ and $q_{1}=0$. (c) Time evolution of modulus of $a_{3}$ for the same initial conditions. (d) Temporal spectrum of modulus of $a_{1}$ for the same initial conditions. The frequency $\omega$ is measured in Hertz. 
a very important difference in the behaviour of the system around the $\mathrm{C} 2$ wave. Before a small perturbation would remain oscillating around C2 with more or less the same amplitude, which means that the waves were stable in the sense that perturbations did not grow. In the present case a small perturbation will grow up to a maximum value and then return to the original state. This is the characteristic phenomenon of the Benjamin \& Feir instability. This recurrence phenomenon represents modulation-demodulation cycles, known as the Fermi--Pasta-Ulam recurrence phenomenon (Fermi et al. 1955). This modulation-demodulation tendency was oberved experimentally by Lake et al. (1977). Numerical solutions of the nonlinear Schrödinger equation (Lake et al. 1977), of the discrete Zakharov equations (Caponi et al. 1982), and of some variants of the Zakharov equations (Shemer \& Stiassnie 1985; Stiassnie \& Shemer 1987) also show the same phenomenon. But all these results show that after a modulation-demodulation period the wave comes back to exactly the initial state. In our model this is only true for the periodic orbit P13; for the rest of the points we have another period which modulates the amplitude of the modulation-demodulation process. This new period was already found by Stiassnie \& Krosynski (1982) using a three-mode model derived from the nonlinear Schrödinger equation. But this new timescale does not appear in the discrete Zakharov equations.

An interesting result which directly follows from the map is that there exist initial conditions such that the new period becomes infinite and the system does not come back to the initial state in finite time. If the initial point is taken lying on the homoclinic orbit to the origin, it will take infinite time for the system to go back to the initial state. This is a solitary wave-like surface. A solution of this kind is shown in figure $10(b-d)$.

Until now we have found quasi-periodic and solitary wave-like solutions, but in all the cases the motion is clearly regular. If we increase the momentum, we find the structure of the map does not change until the momentum reaches the point when the $\mathrm{C} 2$ wave becomes stable again. To study the new region we took $I=0.40$ and considered the motion on the zero energy surface. Again we computed the map by taking several initial points on the $q_{1}=0$ axis. The map that we obtained is shown in figure $11(a)$. We can see that there are three fixed points. From figure 8 it follows that the energy zero surface will contain an orbit from the P13 family and another one from the new P22 family. Besides these two fixed points, we still have the origin as another fixed point. Now, the origin is stable, i.e. a centre, as well as the fixed point corresponding to the P13 family. On the other hand the new fixed point corresponding to the P22 wave is unstable. The origin of the map undergoes a pitchfork bifurcation as it becomes stable. Again because of the degeneracy of the origin we only see one of the two new fixed points. As we will see below, this new fixed point gives rise to a region of irregular motion, i.e. chaotic motion.

Again we have homoclinic connections for the unstable fixed point. From a first look at figure 11(a), it follows that the stable and unstable manifolds coincide and nothing has changed from the previous case, hence the motion is regular in the whole phase space. But this is not true. In figure $11(b)$ we take a closer look at the unstable fixed point. We can very clearly see that there exists a very small region of irregular motion. This suggests that in this case the intersection of the stable and unstable manifolds is transversal. In figure $13(a-d)$ we show the time evolution of the modes $a_{1}$ and $a_{3}$, and the temporal spectrum of $\left|a_{1}\right|$ for initial conditions $p_{1}=-0.162$, $q_{1}=0.0, I=0.4$ and $E=0$.

For $I=0.4$ the region of irregular motion is very small, but it grows as we increase 

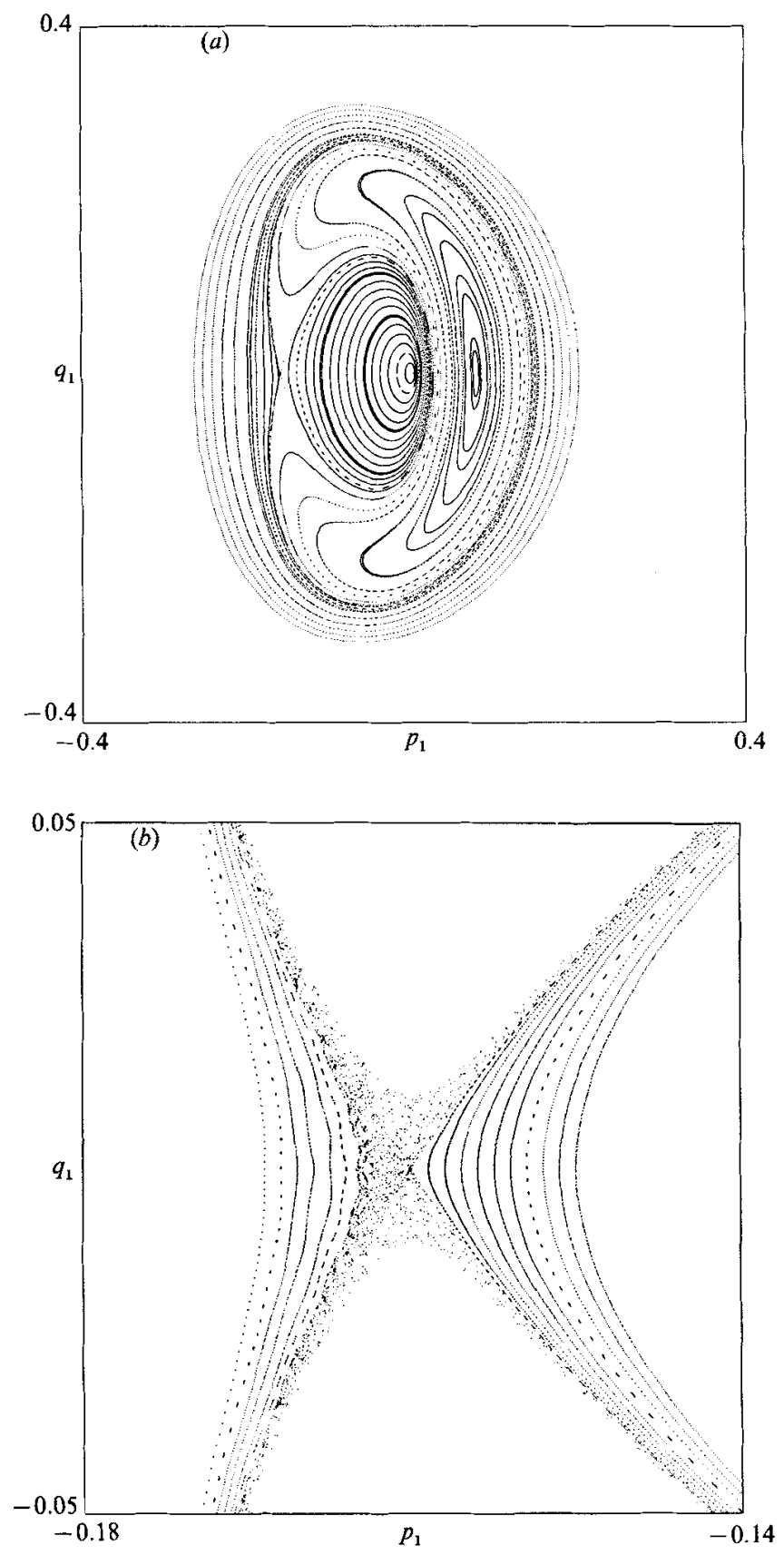

Figure $11(a, b)$. For caption see next page.

I. In figure $12(a-c)$ we show how the chaotic region grows and for large values of the momentum it covers a quite significant region of the $\Sigma_{0}$ surface.

As the momentum is increased the number of periodic orbits start increasing very fast and it becomes impossible to follow all the new branches numerically. This is reflected in the Poincaré maps by the appearance of new fixed points which are centres and are surrounded by small regions within which the motion is regular. 

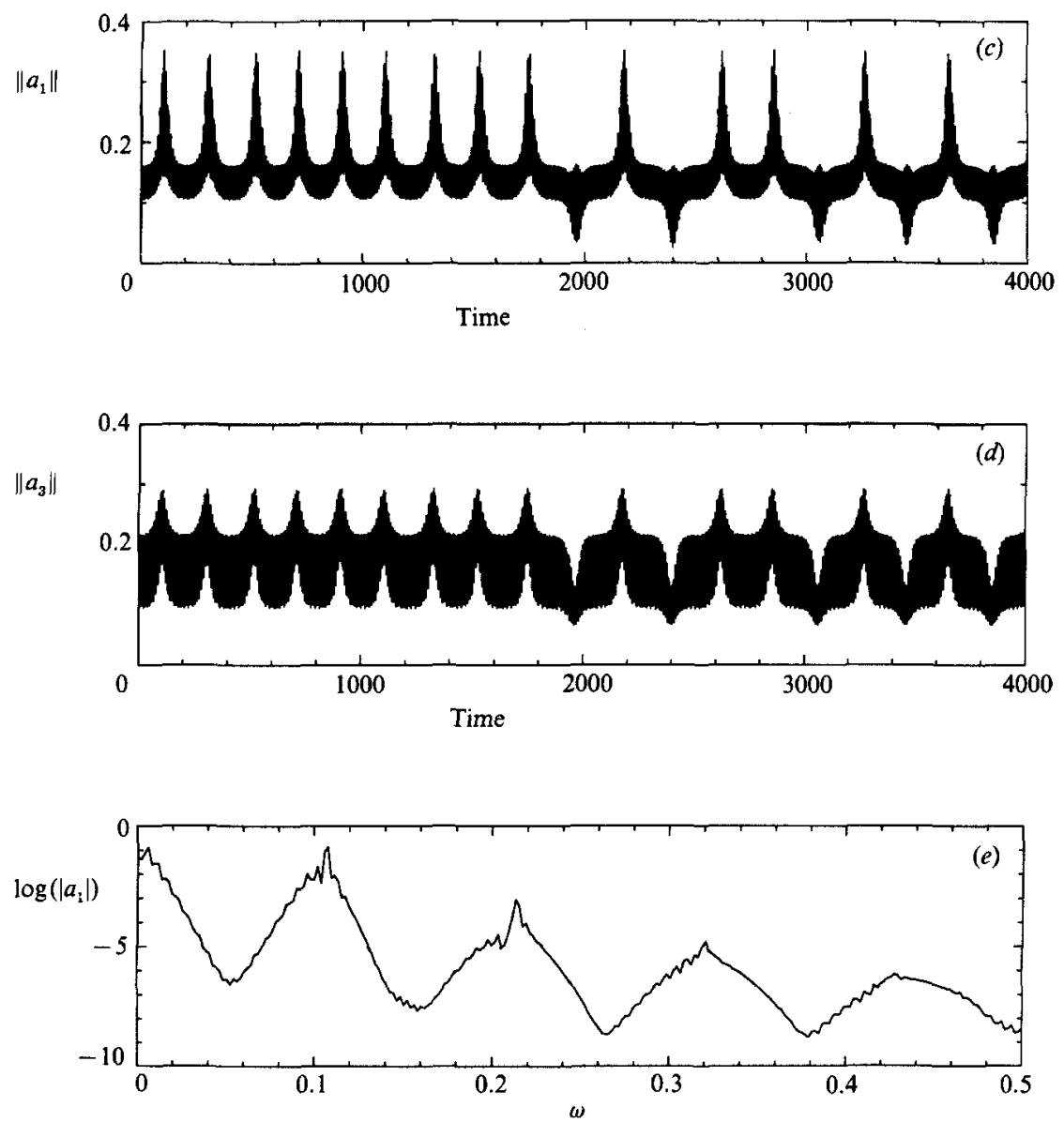

Figure 11. Results for $I=0.4$. (a) Poincare map for the $E=0$ surface. (b) Closer look at the fixed point corresponding to the unstable periodic orbit. Notice chaotic region. (c) Time evolution of modulus of $a_{1}$ for initial conditions $p_{1}=-0.162, q_{1}=0$. (d) Time evolution of modulus of $a_{3}$ for the same initial conditions. (e) Temporal spectrum of modulus of $a_{1}$ for the same initial conditions. The frequency $\omega$ is measured in Hertz.

Some of the bifurcations of the periodic orbits can be observed in the sequence of maps shown in figure $11(a-d)$. For example as we go from $I=1.2$ to $I=1.3$ the stable fixed point which appears in the middle of the chaotic region undergoes a period-doubling bifurcation. We also notice in these maps that there are some regions of regular motion where some isolated points appear. Recall that we are not plotting the true Poincare map on the $\Sigma$ surface, but we are plotting a projection of the map onto the $\left(p_{1}, q_{1}\right)$-plane. This projection is one-to-one for most of the values of the momentum, but when the momentum is high some regions in which the projection is two-to-one appear because of the shape of the $\Sigma$ surface.

In figure $13(a, b)$ we show the time evolution of the mode $a_{1}$ for initial conditions $p_{1}=-0.25, q_{1}=0, I=1$ and $E=0$. Figures $13(c)$ and $13(d)$ show the time evolution and the frequency spectrum of the elevation of the surface $\eta(x, t)$ at $x=0$. From the spectrum it follows that the motion is irregular. This means that if we put a floating probe at a fixed horizontal location the probe will move up and down with chaotic behaviour. 

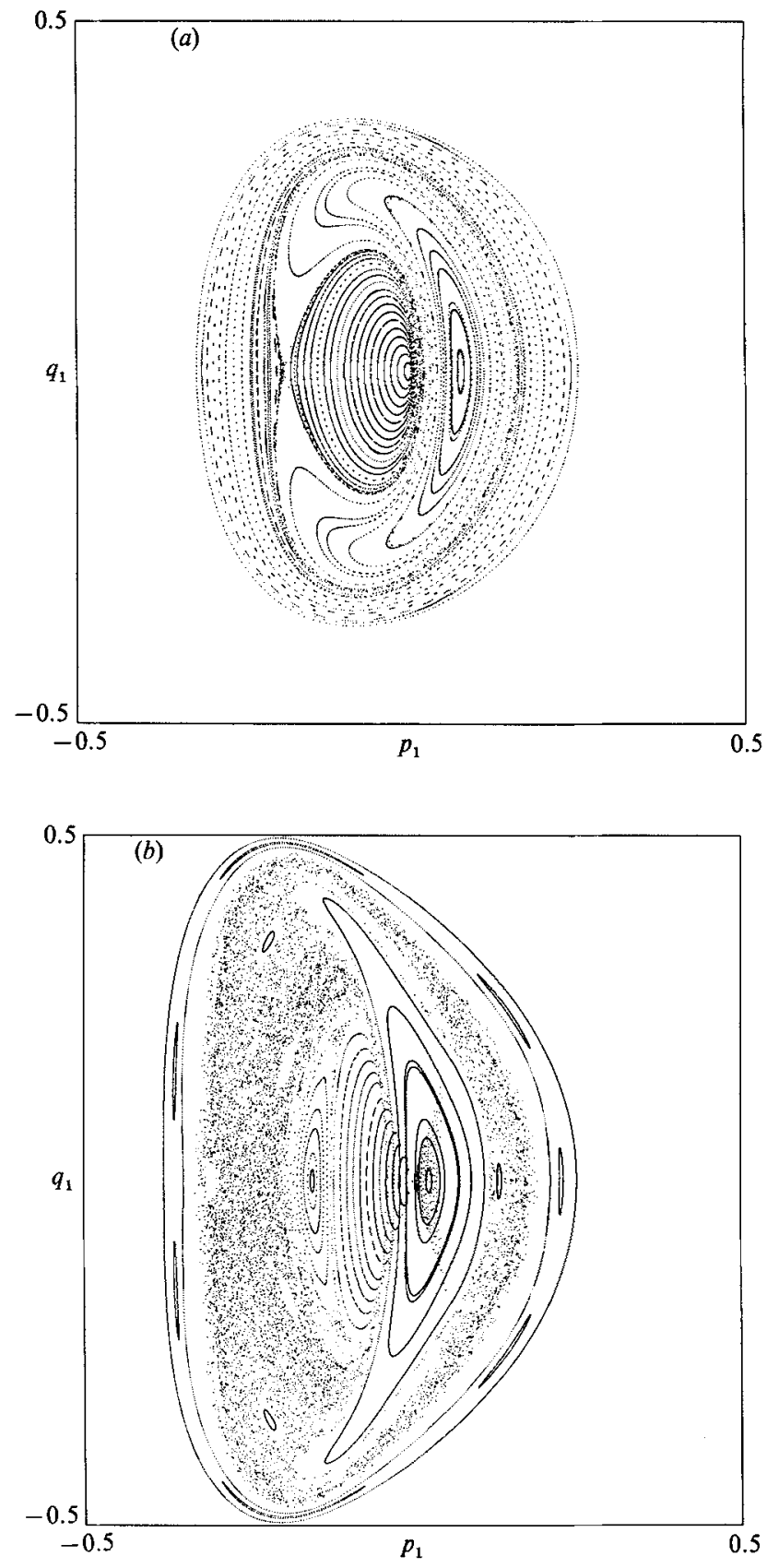

Figure $12(a, b)$. For caption see next page.

All this irregular motion is generated by the loss of stability of periodic orbits which are not necessarily close to the steady C2 wave. In contrast, let us see what happens in the neighbourhood of this fixed point. When $I>0.135$, the fixed point is stable until we reach the period-doubling bifurcation (see figure $3 b$ ). Within this region the two end-points of the P22 family of periodic orbits approach the steady C2 with periods $2 \pi / \omega_{1}$ and $2 \pi / \omega_{3}$. As we approach the period doubling bifurcation, 

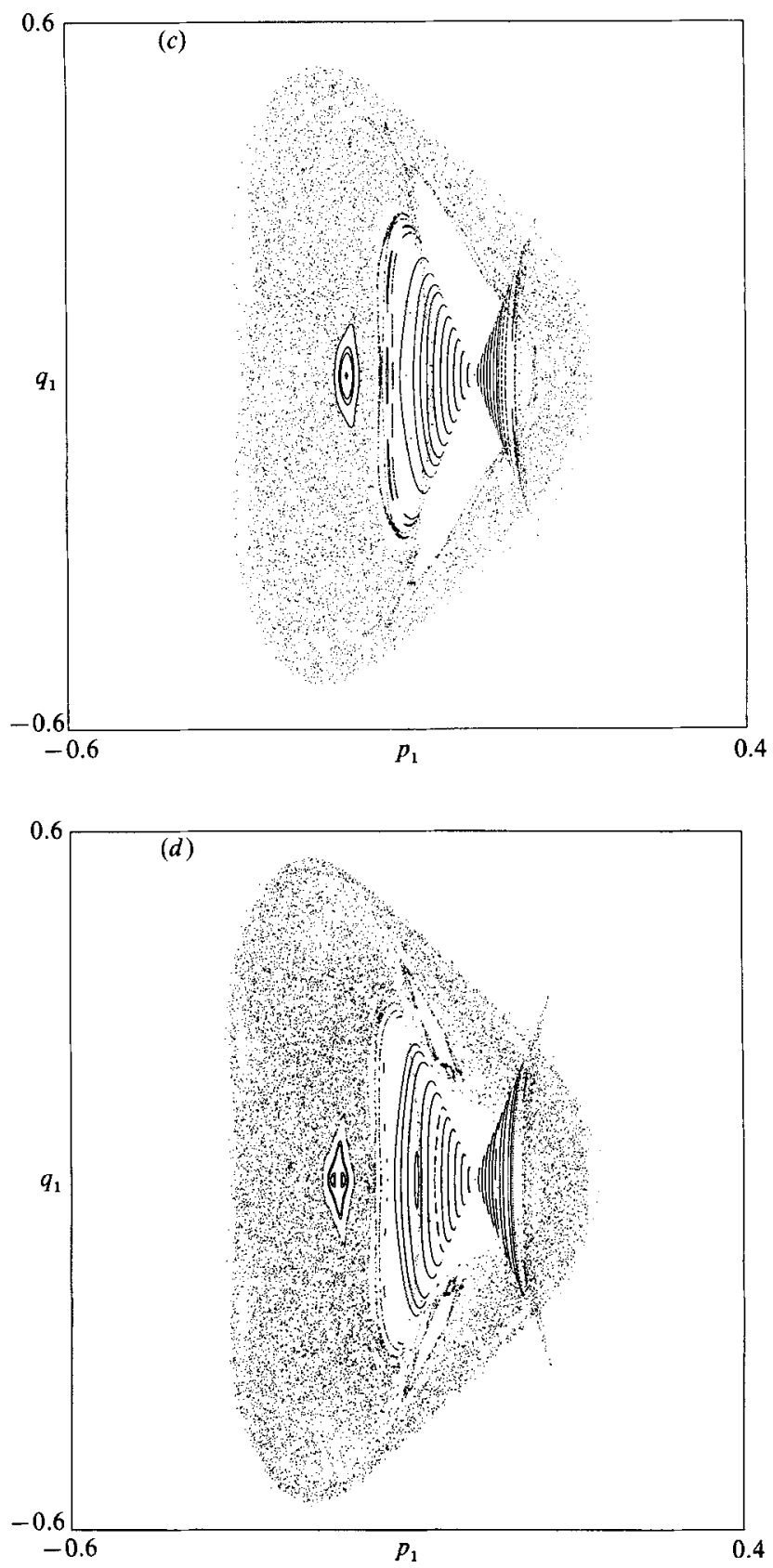

Figure 12. Poincaré maps for the $E=0$ surface for different values of the momentum. (a) $I=0.5$. (b) 1. (c) 1.2. (d) 1.3 .

$\omega_{1} \rightarrow 0$ and the period of the orbit goes to infinity, i.e. close to the period-doubling point there exist periodic orbits with periods arbitrarily large. This in many cases suggests chaotic motion may exist. We have analysed the Poincare map locally for values of the momentum below and above the bifurcation value. We have found that close to the steady $\mathrm{C} 2$ wave the motion is regular when we are below the bifurcation value of $I$. On the other hand, as we cross the bifurcation, a very small region of 
(a)

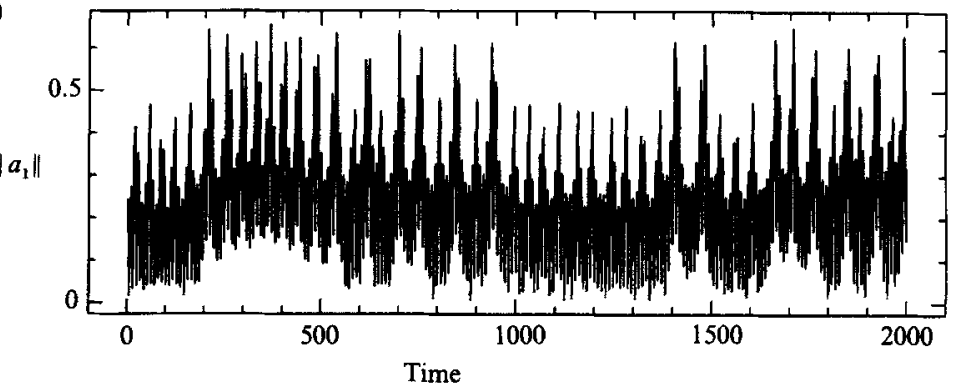

(b)

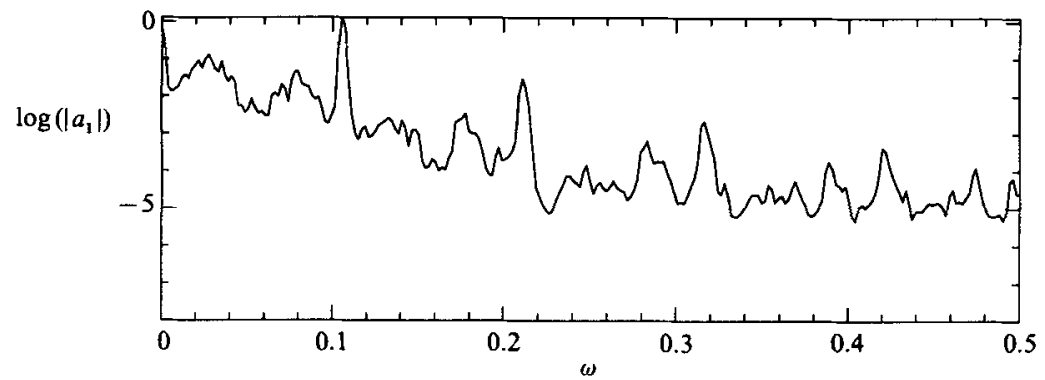

(c)
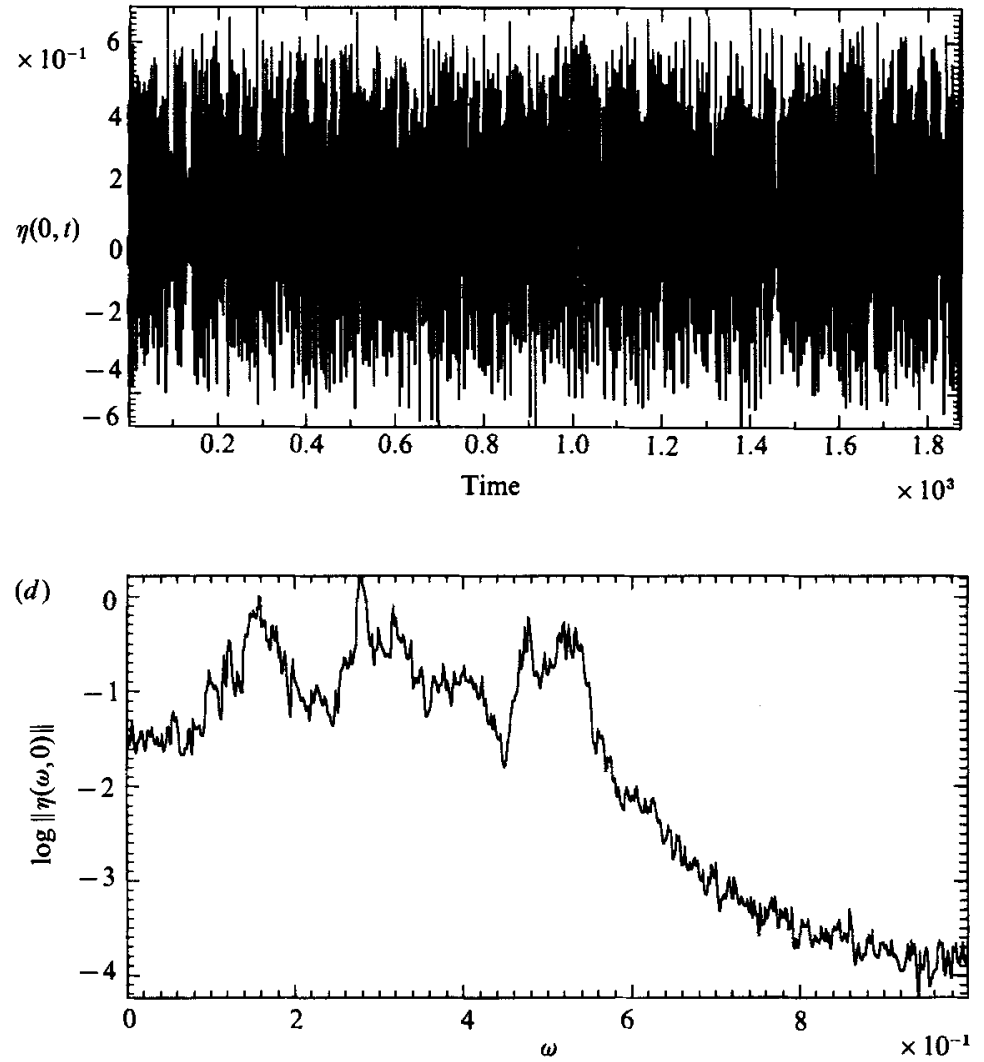

Figure 13. Time evolution for $I=1$ and $E=0$ with initial conditions $p_{1}=-0.25$ and $q_{1}=0$. (a) Modulus of $a_{1}$. (b) Temporal spectrum of modulus of $a_{1}$. (c) Elevation of the surface $\eta(0, t)$. (d) Temporal spectrum of $\eta(0, t)$. The frequency $\omega$ is measured in Hertz. 

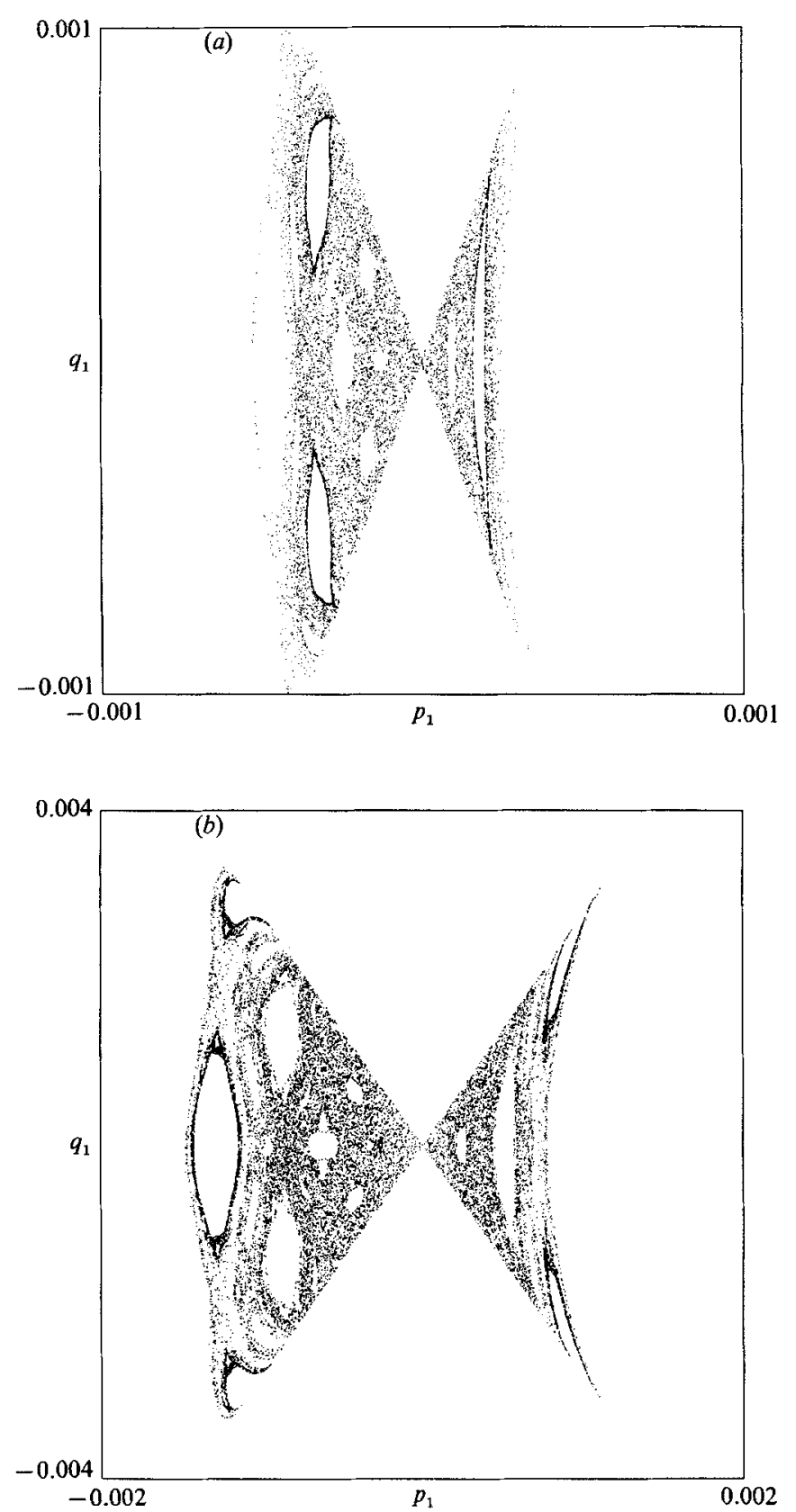

Figure 14. Poincaré map for one orbit close to the origin on the energy $E=0$ surface. (a) $I=1.3$. (b) 1 .4.

irregular motion appears around the steady wave. As the momentum increases this region grows and starts becoming an important part of the phase space. In figure $14(a, b)$, we show the first 20000 iterates of one starting point. These figures suggest that the orbit fills regions of the $\left(p_{1}, q_{1}\right)$-plane which have a fractal dimension larger than one.

The fact that the irregular motion is confined to some angular sectors is again a 


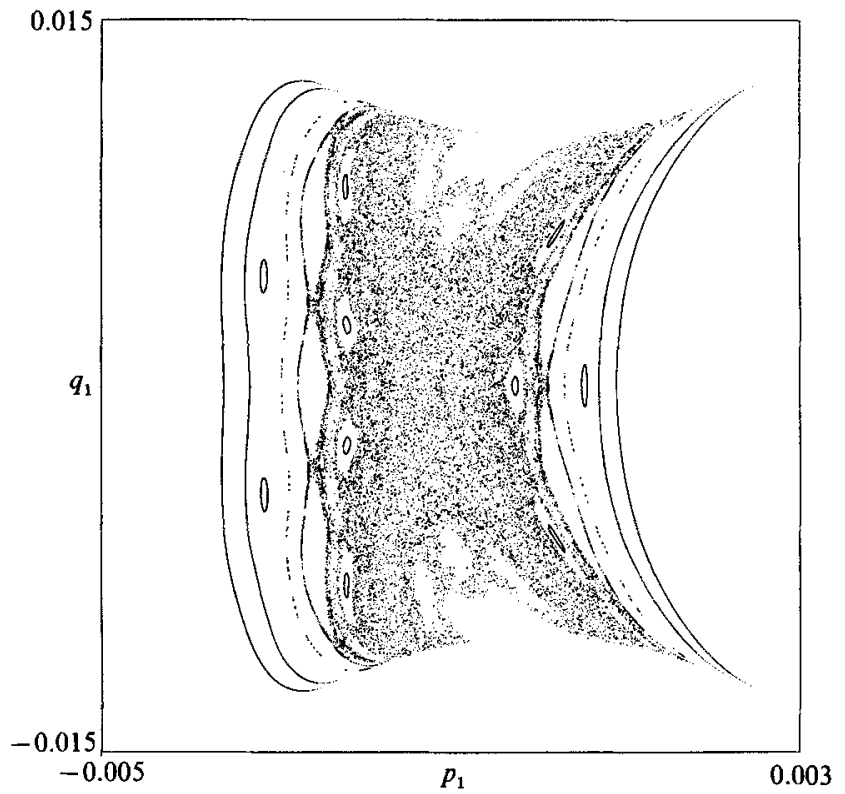

Figure 15. Poincaré map for $I=1.3$ of one orbit lying on the energy $E=0.00001$ surface.

property of the zero-level surface. In figure 15 we show the map for $E=0.00001$. The figure shows that the irregular motion is not confined only to the zero-energy surface. For this energy level, irregular motion does not seem to be confined to an angular sector.

\section{Results and conclusions}

We have used a weakly nonlinear Hamiltonian model to study qualitatively the long-time evolution of gravity water waves. The simplest case for which the model cannot be integrated in closd form has been considered. This leads to a three-freewave model, i.e. a carrier and two-side bands.

Previously, when considering the time evolution of a discrete spectrum of waves, little attention was paid to the idea of constructing models which have the same symmetries as the original equations. Here, special attention has been paid to this point, and we have found that the integrability of our system is in accordance with the symmetries of the original water wave equations.

The model reproduces the steady travelling Stokes waves. It also shows the same superharmonic stability properties as real Stokes waves (for the instabilities which are allowed by the model). From these stability properties, we have shown the existence of a new kind of wave. These new waves have two basic frequencies. The stability of the two-frequency families is considered, and we have found that when these waves are stable the system is expected to undergo quasi-periodic regular motion. In the regions where these solutions are unstable irregular motion is observed.

Considering small perturbations of a steady wave, we have observed that when the motion is regular there are three different timescales. The first one associated to the basic carrier wave. The second one corresponds to a modulation-demodulation period. And the third one modulates the modulation-demodulation period. The last 
timescale cannot be observed by using the discrete Zakharov equations, but it was observed by Stiassnie \& Kroszynski (1982) studying the nonlinear Schrödinger equation.

We have found that chaotic motion appears in two regions of the phase space. Irregular motion is first observed when a new family of two-frequency waves is created in an inverse Hamiltonian Hopf bifurcation. The new unstable wave leads to the existence of stable and unstable manifolds in the phase space which intersect transversally giving rise to a region of chaotic motion. As the momentum of the wave is increased the region of irregular motion grows and fills an appreciable part of the phase space.

The second region of irregular motion appears when the steady class 2 wave loses stability as we cross a period-doubling bifurcation, and a new family of stable steady waves is created. As the bifurcation point is approached the period of one of the twofrequency families goes to infinity creating a homoclinic connection in the phase space. As the bifurcation is crossed a new region of irregular motion appears in the neighbourhood of the unstable class 2 wave.

All the results are obtained using a weakly nonlinear three-wave model. This raises the question of which of these results can be generalized to the infinite dimensional and fully nonlinear water wave system. Comparing our results with the stability results obtained by Longuet-Higgins (1978) with the full water wave equations, we expect, going from the weakly nonlinear case to the fully nonlinear, changes in the amplitudes at which the phenomena occur, but no qualitative changes are expected. This is because most of the results that we have obtained are due to the behaviour of pairs of eigenvalues of a Hamiltonian system and the behaviour of the eigenvalues of our system is similar to that found by Longuet-Higgins (1978) with the full equations.

In relation to the number of modes of the system, we believe that, even though numerical simulation with more modes needs to be done and will be the subject of a future study, most of the results are not related to the dimension of the phase space and we think they can be generalized to the full infinite dimensional system. For example, the existence of the two-frequency waves is a property related to the character of the eigenvalues of the superharmonic stability of the Stokes waves. Notice that according to the Liapunov theorem the existence of a family of twofrequency waves is related only to the existence of a pair of pure imaginary eigenvalues. As more modes are considered more families will exist because more eigenvalues are added to the system, but families will not disappear. We have seen that the stability properties of the steady waves are not related to the number of modes of the system, therefore we may expect that this will be also the case for the two-frequency families. Also, the behaviour of the two-frequency families as we cross the Hamiltonian Hopf bifurcation will happen in the same way in a four-dimensional manifold contained within the infinite dimensional phase space.

Finally, as we approach the period-doubling bifurcation, which is known to exist in the full equations, one of the periods of the two-frequency waves will go to infinity and will lead to a homoclinic orbit in the infinite dimensional phase space. Hence, we also expect irregular motion in the full equations when motion close to the period doubling bifurcation is considered.

Numerical simulations with the full equations are very expensive because the system has to be run for very long time periods to observe the different motions that we described above. However, we hope that numerical simulations with the full equations will be performed to verify the above statements. 
I am indebted to Professor P. G. Saffman for his encouragement and valuable advice during the course of the research. This work was supported by the Office of Naval Research (N00014-79-C-0412, NR062-639) and by the National Science Foundation (OCE-8415988). I also wish to acknowledge receipt of an award from the Comité Conjunto Hispano-Norteamericano para la cooperacion cultural y educativa.

\section{REFERENCES}

Abrahay, R. \& Marsden, J. E. 1978 Functions of Mechanics, 2nd edn. Benjamin/Cummings, Massachusetts.

ARnold, V.I. 1978 Mathematical Methods of Classical Mechanics. Springer.

Benja min, T. B. 1984 Impulse, Force and Variational Principles. IMA J. Appl. Maths 32, 368.

Benjamin, T. B. \& Feir, J. E. 1967 The disintegration of wave trains in deep water. Part 1. Theory. J. Fluid Mech. 27, 417-430.

Broer, L. J. F. 1974 On the Hamiltonian theory of surface waves. Appl. Sci. Res. 29, 430446.

Brown, E. W. 1911 On the oscillating orbits about the triangular equilibrium points in the problem of three bodies. Mon. Not. R. Astr. Soc. 71, 492-502.

Buchanan, D. 1941 Trojan satellites (limiting case). Trans. R. Soc. Canada §3, 3, 9-25.

Caponi, E. A., SAffman, P. G. \& Yuen, H. C. 1982 Instability and confined chaos in a nonlinear dispersive wave system. Phys. Fluids 25, 2159-2166.

Chen, B. \& Saffman, P. G. 1980 Numerical evidence for the existence of new types of gravity waves of permanent form on deep water. Stud. Appl. Maths 62, 1-21.

Devaney, R. L. 1976 Homoclinic orbits in Hamiltonian systems. J. Diff Equat. 21, 431-438.

Doedel, E. J. \& Kernevez, J. P. 1986 Software for continuation problems in ordinary differential equations with applications. Coltech Appl. Maths Rep.

Fermi, E., Pasta, J. \& Ulam, S. 1955 Studies of nonlinear problems. In Collected Papers of Enrico Fermi, vol. 2, p. 978. University of Chicago Press, 1962.

Goldstein, H. 1980 Classical Mechanics, 2nd edn. Addison Wesley.

Green, J. M., MacKay, R. S., Vivaldi, F. \& Feigenbaum, M. J. 1981 Universal behavior in families of area preserving maps. Physica $3 \mathrm{D}, \mathbf{4 6 8 - 4 8 6 .}$

Guckenheimer, J. \& Holmes, P. 1983 Nonlinear Oscillations, Dynamical Systems and Bifurcations of Vector Fields. Springer.

Hasimoto, H. \& Ono, H. 1972 Nonlinear modulation of gravity waves. J. Phys. Soc. Japan 33, 805-811.

JACOBI, C. G. J. 1836 Sur le mouvement d'un point et sur un cas particulier du problème des trois corps. C. R. Acad. Sci. Paris 3, 59.

Lake, B., Yuev, H. C., Rungaldier, H. \& Ferguson, W. E. 1977 Nonlinear deep-water waves: theory and experiments. Part 2. Evolution of a continuous wave train. J. Fluid Mech. 83, $49-74$.

LeRMan, L. M. \& Umanskit, I. L. 1984 On the existence of separatrix loops in four-dimensional systems similar to the integrable Hamiltonian systems. Physics Metals Metallogr. USSR 47, no. 3, 335-340.

LighthilL, M. J. 1965 Contributions to the theory of waves in nonlinear dispersive systems. J. Inst. Math. Appl. 1, 269.

LoNGUET-Higarns, M. S. 1978 The instabilities of gravity waves of finite amplitude in deep water. II. Subharmonics. Proc. R. Soc. Lond. A 360, 489-505.

Longuet-Higgins, M. S. 1985 Bifurcation in gravity waves. J. Fluid Mech. 151, 457-475.

Lonavet-Hiagins, M. S. 1986 Bifurcation and instability in gravity waves. Proc. R. Soc. Lond. A 403, 167-187.

MacKay, R.S. $1986 a$ Stability of equilibria of Hamiltonian systems. In Nonlinear Phenomena and Chaos (ed. S. Sarkar), Adam Hilger. 
MacKay, R. S. $1986 b$ Introduction to the dynamics of area-preserving maps. Proceeding of the US Particle Accelerator School SLAC 1985 (ed. M. Month).

MacKay, R. S., Meiss, J. D. \& Percival, I. C. 1984 Transport in Hamiltonian systems. Physica $13 \mathrm{D}, 55-81$.

Mackay, R. S. \& Saffman, P. G. 1986 Stability of water waves. Proc. R. Soc. Lond. A 406, $115-125$.

Olver, P. J. 1986 Applications of Lie Groups to Differential equations. Springer.

Poincaré, H. 1985 Sur les courbes défines par les équations différentielles. J. Math. Pure Appl. ser. 4, 1, 167-244.

Porncaré, H. 1890 La problème des trois corps et les equations de la dynamique. Acta Math. 13, $1-270$.

Routh, E. J. 1875 On Laplace's three particles, with a supplement on the stability of steady motion. Proc. Lond. Math. Soc. 6, 86-97.

SAFFMAN, P. G. 1980 Long wavelength bifurcation of gravity waves on deep water. J. Fluid Mech. $101,567-581$.

Saffman, P. G. 1985 The superharmonic instability of finite-amplitude water waves. J. Fluid Mech. 159, 169-174.

Shemer, L. \& Strassnie, M. 1985 Initial instabilities and long-time evolution of Stokes waves. The Ocean Surface: Wave Breaking, Turbulent Mixing and Radio Probing (ed. Y. Toba \& $\mathbf{H}$. Mitsuyasu), pp. 51-57. D. Reidel.

Stiassnie, M. \& Kroszynski, U. I. 1982 Long-time evolution of an unstable water-wave train. J. Fluid Mech. 83, 49-74.

Stiassnie, M. \& Shemer, L. 1987 Energy computations for modulations of class I and II instabilities of Stokes wave. J. Fluid Mech. 174, 299-312.

VAN der Meer, J. C. 1985 The Hamiltonian Hopf Bifurcation. Lectures Notes on Physics, 1160, Springer.

Van der Megr, J. C. 1986 Bifurcation at nonsemisimple $1:-1$ resonance. $Z$. angew. Math. Phys. $37,425-437$.

YUEN, H. C. 1984 Order and chaos in the long-time evolution of a nonlinear wave train. Symposium on Wave Breaking, Turbulent Mixing, and Radio Probing of the Ocean Surface. Sendai, Japan (unpublished contribution).

Yuen, H. C. \& LAKe, B. M. 1982 Nonlinear dynamics of deep-water gravity waves. Adv. Appl. Mech. 22, 67-229.

ZAKHAROV, V. E. 1968 The instability of periodic waves of finite amplitude on the surface of deep fluid. J. Appl. Mech. Tech. Phys. 2, 190-194.

ZUFIRIA, J. A. 1987 a Weakly nonlinear nonsymmetric gravity waves on water of finite depth. J. Fluid Mech. 180, 371-385.

ZufiRia, J. A. $1987 b$ Nonsymmetric gravity waves on water of infinite depth. J. Fluid Mech. 181, 17-39.

ZUFIRIA, J. A. 1987 c Symmetry breaking in periodic and solitary gravity-waves on water of finite depth. J. Fluid Mech. 184, 183-206.

Zufiria, J. A. \& Saffman, P. G. 1986 The superharmonic instability of finite amplitude surface waves on water of finite depth. Stud. Appl. Maths 74, 259-266. 